\title{
Intrinsic Firing Dynamics of Vestibular Nucleus Neurons
}

\author{
Chris Sekirnjak and Sascha du Lac \\ Systems Neurobiology Laboratories, The Salk Institute for Biological Studies, La Jolla, California 92037
}

Individual brainstem neurons involved in vestibular reflexes respond to identical head movements with a wide range of firing responses. This diversity of firing dynamics has been commonly assumed to arise from differences in the types of vestibular nerve inputs to vestibular nucleus neurons. In this study we show that, independent of the nature of inputs, the intrinsic membrane properties of neurons in the medial vestibular nucleus substantially influence firing response dynamics. Hyperpolarizing and depolarizing inputs evoked a markedly heterogenous range of firing responses. Strong postinhibitory rebound firing (PRF) was associated with strong firing rate adaptation (FRA) and occurred preferentially in large multipolar neurons. In response to sinusoidally modulated input current, these neurons showed a pronounced phase lead with respect to neurons lacking strong PRF and FRA. A combination of the hyperpolarization-activated $\mathrm{H}$ current and slow potassium currents contributed to PRF, whereas FRA was predominantly mediated by slow potassium currents. An integrate-and-firetype model, which simulated FRA and PRF, reproduced the phase lead observed in large neurons and showed that adaptation currents were primarily responsible for variations in response phase. We conclude that the heterogeneity of firing dynamics observed in response to head movements in intact animals reflects intrinsic as well as circuit properties.

Key words: vestibular nucleus neuron; spike frequency adaptation; postinhibitory rebound; $\mathrm{I}_{H}$; potassium current; phase lead
To produce accurate behavioral responses, neural circuits must provide output signals with appropriate temporal properties. The vestibulo-ocular reflex (VOR) is well suited for the study of temporal signal dynamics because the underlying neurons and the control signals they carry have been studied extensively. In response to head movement, the VOR produces eye movements that rapidly and accurately stabilize images on the retina. To ensure faithful image stability over the wide range of behaviorally relevant head movement frequencies, the neural circuitry for the VOR must transform head movement inputs into the precise command signals to ocular motoneurons that are required to move the eye accurately while compensating for phase lags imposed by orbital mechanics (Skavenski and Robinson, 1973; Highstein, 1988).

The temporal processing requirements of the vestibular system are reflected in the diversity of neuronal firing responses to head movements. Neurons in the vestibular nuclei respond to identical sinusoidally modulated head movements with a wide range of response phases that vary over many tens of degrees across neurons (Shinoda and Yoshida, 1974; Fuchs and Kimm, 1975; Buettner et al., 1978; Lisberger and Miles, 1980; Scudder and Fuchs, 1992). It is commonly assumed that such variations in the phase of firing responses evoked by sinusoidal head movements are conferred by differences in the types of vestibular nerve afferents synapsing onto distinct types of vestibular nucleus neurons (Keller and Precht, 1979; Lisberger and Miles, 1980; Baker et al., 1984; Kasper et al., 1988; Wilson et al., 1990; Endo et al.,

\footnotetext{
Received Aug. 22, 2001; revised Dec. 20, 2001; accepted Dec. 28, 2001.

This work was supported by National Institutes of Health Grant EY11027 and by a fellowship from the Sloan Center for Theoretical Neurobiology at the Salk Institute (C.S.). We thank Edward Callaway, Jeffrey Isaacson, Richard Krauzlis, and Alexandra Nelson for their valuable comments.

Correspondence should be addressed to Sascha du Lac, SNL-D, The Salk Institute for Biological Studies, 10010 North Torrey Pines Road, La Jolla, CA 92037. E-mail: sascha@salk.edu.

Copyright (ㄷ) 2002 Society for Neuroscience $\quad 0270-6474 / 02 / 222083-13 \$ 15.00 / 0$
}

1995). This assumption has arisen primarily from the observation that vestibular nerve afferents themselves exhibit a wide range of temporal response patterns during head movements (Fernandez and Goldberg, 1971; Keller, 1976; Louie and Kimm, 1976; Ezure et al., 1978; Anastasio et al., 1985; Boyle and Highstein, 1990). However, firing responses depend not only on synaptic input signals but also on filtering imposed by intrinsic membrane currents. In many systems, biophysical properties of neurons have significant influences on the firing responses to input signals (for review, see Llinas, 1988; Destexhe et al., 1996; Oertel, 1997). Little is known, however, about how voltage- and time-dependent ionic currents contribute to temporal dynamics of firing and the diversity of response properties observed in vestibular nucleus neurons.

Although membrane properties of vestibular nucleus neurons have been studied in a number of species, most investigations have focused on action potential shapes, spontaneous firing, and subthreshold membrane currents (Serafin et al., 1991a,b; Johnston et al., 1994; du Lac and Lisberger, 1995a). On the basis of the afterhyperpolarizations following each action potential, vestibular nucleus neurons have been classified into two types (Serafin et al., 1991a; Johnston et al., 1994), which appear to form a continuous population (du Lac and Lisberger, 1995a; Babalian et al., 1997). Analyses of intrinsic response properties of medial vestibular nucleus (MVN) neurons have focused on linearity (du Lac and Lisberger, 1995b) and gain (Ris et al., 2001; Smith et al., 2002). Although these studies indicate that vestibular neurons are, in fact, heterogenous with respect to intrinsic ionic currents, they do not address whether and how these currents confer variations in firing response phase.

To determine how intrinsic firing properties could contribute to the range of response phases observed in vivo, we analyzed the firing response dynamics of MVN neurons recorded with wholecell patch electrodes in brain slices. Using synaptic stimulation 
and intracellular current injection, we found that the intrinsic firing responses to hyperpolarizing, depolarizing, and sinusoidally modulated stimuli vary widely across MVN neurons. Pharmacological and computational analyses revealed that variations in response dynamics are conferred by at least two types of ionic currents that depend on previous membrane potential and firing. Our results indicate that ionic currents shape the dynamics of MVN neuronal responses to sensory stimuli.

\section{MATERIALS AND METHODS}

Slice preparation. Slices were prepared from 14- to 19-d-old mice of mixed C57BL/6 and BALB/c background (mean age, $17 \mathrm{~d}$ ). The animals were deeply anesthetized with sodium pentobarbital and decapitated. The hindbrain was rapidly removed from the skull and placed in ice-cold artificial CSF (ACSF) aerated with $95 \% \mathrm{O}_{2}$ and $5 \% \mathrm{CO}_{2}$. A tissue block containing the brainstem with attached cerebellum was dissected away and glued to a Teflon chuck with cyanoacrylate. Coronal slices of $300 \mu \mathrm{m}$ thickness were prepared on a vibratome (Campden Instruments, Lafayette, IN) in ice-cold aerated ACSF. All slices were cut at a level rostral to the dorsal cochlear nucleus and caudal to the root of the facial nerve. The slices were transferred to a holding chamber and incubated for at least $1 \mathrm{hr}$ at room temperature in aerated ACSF.

Electrophysiology. The bath solution (ACSF) contained (in $\mathrm{mM}$ ): 124 $\mathrm{NaCl}, 5 \mathrm{KCl}, 1.3 \mathrm{MgSO}_{4}, 26 \mathrm{NaHCO}_{3}, 2.5 \mathrm{CaCl}_{2}, 1 \mathrm{NaH}_{2} \mathrm{PO}_{4}$, and 11 dextrose. The glutamate receptor blocker kynurenic acid (2 mM) was added to the bath solution in all experiments. Aerated ACSF had a final $\mathrm{pH}$ of 7.4 and an osmolarity of $300 \mathrm{mOsm}$. For ACSF containing zero $\mathrm{Ca}^{2+}, \mathrm{CaCl}_{2}$ was replaced with equimolar $\mathrm{MgCl}_{2}$; for low-K ${ }^{+}$bath solutions, $\mathrm{KCl}$ was reduced from 5 to $1 \mathrm{~mm}$.

Micropipettes for whole-cell recordings were fabricated from borosilicate glass (outer diameter $1.5 \mathrm{~mm}$; Garner Glass, Claremont, CA) with a Sutter Instruments (Novato, CA) P-97 puller and had resistances of 5-15 $\mathrm{M} \Omega$. The internal recording solution contained (in $\mathrm{mM}$ ): 140 K-gluconate, $8 \mathrm{NaCl}$, $10 \mathrm{HEPES}, 0.1 \mathrm{EGTA}, 2 \mathrm{Mg}$-ATP, and $0.3 \mathrm{Na}-$ GTP. The $\mathrm{pH}$ was adjusted to $7.2-7.5$ and the osmolarity to $280-285$ mOsm.

Whole-cell patch recordings were performed in a submersion-type chamber with continuous perfusion of aerated ACSF. Neurons were visualized at $40-80 \times$ with an infrared differential interference contrast (DIC) microscope (Olympus, Tokyo, Japan) at depths of 30-90 $\mu \mathrm{m}$ below the slice surface. Recordings were made with a AxoClamp 2B amplifier (Axon Instruments, Foster City, CA) in current-clamp mode. Access resistance was checked and compensated regularly throughout each experiment. Input resistance was measured by injecting small hyperpolarizing current pulses from a membrane potential of approximately $-80 \mathrm{mV}$. Signals were filtered at $10 \mathrm{kHz}$, digitized at $2-20 \mathrm{kHz}$, and recorded using Macintosh G3 and G4 computers. Intracellular current injection, as well as triggering of the extracellular stimulating electrode, were controlled by the computer. All recordings were made at a temperature of $31-33^{\circ} \mathrm{C}$. A calculated liquid junction potential of 14 $\mathrm{mV}$ was subtracted from all membrane potentials.

Monopolar tungsten electrodes (FHC Inc, Bowdoinham, ME) were used for extracellular stimulation of axons. The electrode was placed at a distance of $200-800 \mu \mathrm{m}$ from the recorded neuron, usually near the dorsolateral rim of the MVN. This region contains the axons of cerebellar Purkinje cells projecting to the vestibular nuclei (Brueckner et al., 2001) (C. Sekirnjak and S. du Lac, unpublished observations). Currents of $50-200 \mu \mathrm{A}$ were applied to elicit inhibitory postsynaptic potentials.

Kynurenic acid, cesium chloride, tetrodotoxin, and cadmium chloride were obtained from Sigma (St. Louis, MO), and ZD7288 was obtained from Tocris Cookson (Ballwin, MO). For dye filling of cells, tetramethylrhodamine dextran (Molecular Probes, Eugene, OR) at $0.1 \mathrm{mg} / \mathrm{ml}$ was included in the internal recording solution. The slices were fixed overnight in $4 \%$ paraformaldehyde, frozen, and resectioned at $100 \mu \mathrm{m}$. Images were acquired on a Olympus fluorescence microscope using a Hamamatsu (Tokyo, Japan) digital camera. To capture all neuronal processes, several focal planes were imaged and superimposed in Adobe Photoshop (Adobe Systems, San Jose, CA).

Data analysis. The recorded signals were analyzed offline using IgorPro software (Wavemetrics, Lake Oswego, OR). Instantaneous firing rate was calculated as the reciprocal of the interval between successive spikes and was assigned to the time of the second spike. The width of the action potential was measured at spike threshold, and the afterhyperpo- larization (AHP) amplitude was calculated as the membrane potential difference between spike threshold and the absolute membrane potential minimum after the falling phase of the spike. Both values were calculated from averages of $10-20$ action potentials, aligned at the peak. For the calculation of spike threshold, see Murphy and du Lac (2001). Postinhibitory rebound firing (PRF) was defined as the difference in firing rate before and immediately after a 1 sec hyperpolarizing current pulse. Membrane sag was defined as the difference between the membrane potential at the initial peak and the end of a $1 \mathrm{sec}$ hyperpolarizing current pulse; measurements were made on the same data traces used to quantify $\mathrm{PRF}$ in each neuron. Firing response gains were calculated by injecting depolarizing $1 \mathrm{sec}$ current steps of increasing amplitude and calculating the mean firing rate during each step. Because most MVN neurons show linear current-firing rate relationships (du Lac and Lisberger, 1995a,b), the slope of a best-fit line was used to define response gain.

Firing rate adaptation (FRA) was quantified as either the difference (absolute adaptation) or the ratio of $100 \mathrm{msec}$ averages of firing rate at the beginning and the end of each current step. The decay in firing rate could be fit well by a single exponential beginning $50 \mathrm{msec}$ after step onset (thereby excluding the first few interspike intervals, which varied markedly across step amplitudes and neurons). Therefore, we excluded the first $50 \mathrm{msec}$ from analyses of adaptation. To standardize the analyses of adaptation across neurons, analyses were restricted to firing responses of $<40$ spikes/sec at the end of the step.

Sinusoidal functions $f(t)=A+B \sin (2 \pi \mathrm{ft}+\mathrm{C})$ were fitted to the firing rate responses to sinusoidally oscillating current injections, with the parameters $A, B$, and $C$ chosen to minimize the mean squared error. The frequency $f$ was set equal to the input frequency. Phase shifts were given by the best-fit values of parameter $C$. A stretch of at least four or five cycles of each frequency was used to obtain fits. For firing rate responses that displayed cutoff during a half-cycle, data point sequences between silent periods were fitted individually, and the resulting fit parameters were averaged. To estimate the goodness of sinusoidal fits, firing rate values predicted by the best sine fit were regressed against actual firing rate values and the linear correlation coefficients determined. Slopes for these regression lines were always close to 1 , with intercepts near 0 . Values for $R^{2}$ ranged from 0.95 to 0.99 .

To estimate cell size, DIC images of neurons acquired during wholecell recordings were analyzed. Each cell shape was approximated by an ellipse, and the product of the long and the short axis was used as the approximate neuronal somatic area. The number of primary processes was counted in the living slices immediately after dye filling, or in paraformaldehyde fixed slices after resectioning.

ANOVA (StatView software) was used to analyze group differences in mean input resistance, spike width, AHP, gain, and phase shifts for neurons with weak, medium, and strong rebound. Post hoc comparisons were made using tests that do not assume equal variances of the groups (Scheffé). A $p$ value $<0.05$ was considered significant. All error values given are SEMs, unless otherwise indicated. All values of $n$ stated are numbers of neurons.

Neuronal model. A computer model of firing rate dynamics was implemented in IgorPro. It consisted of an leaky integrate-and-fire algorithm (Knight, 1972) that incorporated additional conductances to simulate PRF and FRA. Briefly, we used an electrical membrane model that calculates changes in membrane potential from the underlying ionic conductances and an external current. Whenever the membrane voltage exceeds a spike threshold, a spike of fixed amplitude and duration is generated. For a given time step $\Delta t$, the subthreshold membrane potential change was calculated as:

$$
\Delta V=\frac{I(t)}{C} \Delta t \quad \text { with } \quad I(t)=\sum_{i} g_{\mathrm{i}}\left(V_{\mathrm{i}}-V(t)\right)+I_{\text {leak }}(t)+I_{\text {input }}(t),
$$

where $I(t)$ is the total current, $C$ the input capacitance $(C=0.1), \Delta t$ the time step increment $(\Delta t=100 \mu \mathrm{sec}), I_{\text {leak }}$ the leak current, and $I_{\text {input }}$ the input current (steps or sinewaves). Each time spike threshold was reached, an action potential was simulated by setting the membrane voltage to $15 \mathrm{mV}$, followed by a reset to $-60 \mathrm{mV}$. In each simulation, the current injection was preceded by $10 \mathrm{sec}$ of spontaneous or evoked firing, during which all conductances reached equilibrium.

To describe firing response variations in MVN neurons, two conductances were used: a rebound conductance $g_{P R F}$ and an adaptation conductance $g_{F R A}$. The PRF conductance activated exponentially with membrane hyperpolarizations below an activation potential $V_{\text {act }}$ 
A

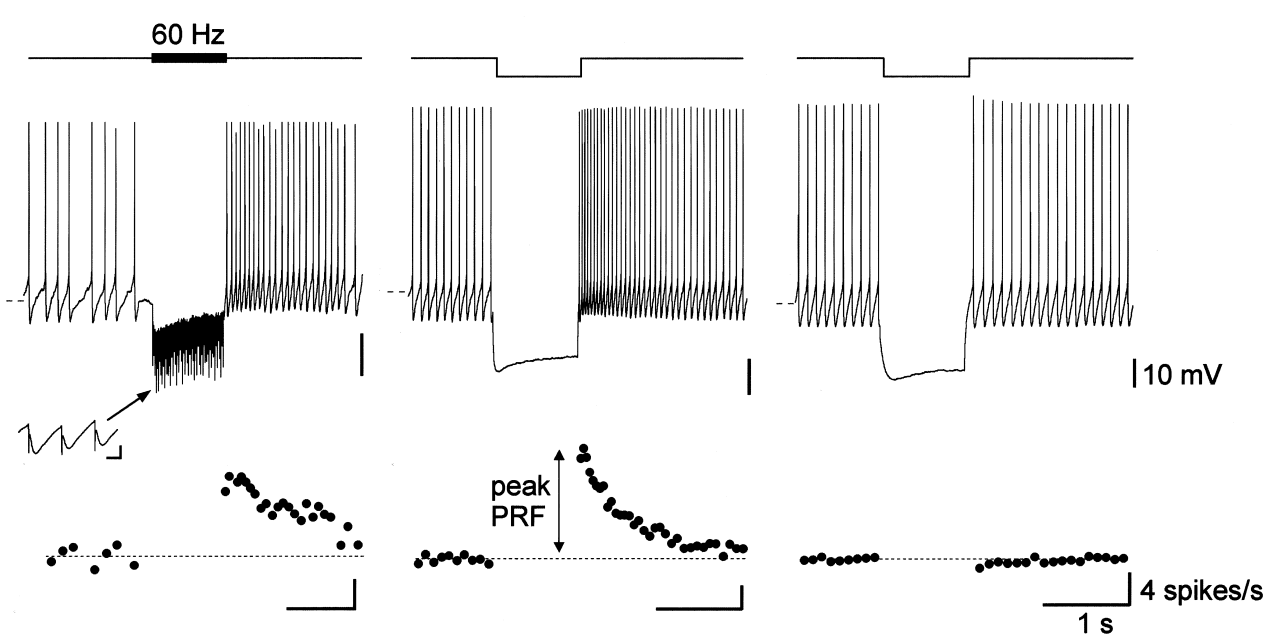

Figure 1. Postinhibitory rebound firing in MVN neurons. Traces show membrane potential as a function of time in response to synaptic stimulation $(A)$ or current injection $(B, C)$ in three different neurons. The corresponding instantaneous firing rates are plotted below. $A$, Synaptically evoked rebound firing, induced by a $60 \mathrm{~Hz}$ stimulus train. Inset shows individual IPSPs during stimulation on an expanded scale. Calibration: $2 \mathrm{mV}, 20 \mathrm{msec}$. Dotted line in bottom trace indicates spontaneous firing rate ( 5 spikes/sec). $B$, Rebound firing evoked by intracellular injection of a -200 pA current pulse (top trace). The double arrow in the bottom trace indicates the definition of peak PRF. Spontaneous firing rate, 11 spikes/sec. $C$, Example of a neuron that displayed no rebound firing in response to a $-100 \mathrm{pA}$ current injection. Spontaneous firing rate, 9 spikes/sec. Dashed lines in top traces indicate $-55 \mathrm{mV}$ in $A$ and $-60 \mathrm{mV}$ in $B$ and $C$.

$$
\Delta g_{\text {PRF }}=\Delta g_{\text {PRFmax }} \cdot e^{-\frac{\lambda}{V_{\text {act }}-\mathrm{V}}}
$$

with $\mathrm{V}_{\text {act }}=-15 \mathrm{mV}, \lambda=70 \mathrm{mV}$. Using this implementation, $10 \%$ activation was achieved at approximately $-45 \mathrm{mV}$ and $\sim 40 \%$ with hyperpolarizations to $-90 \mathrm{mV}$. The maximum rebound conductance $\Delta g_{\text {PRFmax }}$ was $2.5 * 10^{-6}$ for neuron A and $3 * 10^{-7}$ for neuron B. The adaptation conductance was increased by a fixed amount $\Delta \mathrm{g}_{F R A}$ after every spike: $1.63 * 10^{-5}$ for neuron $\mathrm{A}$ and $1.63 * 10^{-6}$ for neuron $\mathrm{B}$. Both conductances were set to decay exponentially during the interspike interval with time constants of $\tau_{\mathrm{PRF}}=300$ and $1000 \mathrm{msec}$ for rebound in neuron $\mathrm{A}$ and $\mathrm{B}$, respectively, and $\tau_{\mathrm{FRA}}=1500 \mathrm{msec}$ for adaptation in both neurons. The corresponding currents were modeled with equilibrium potentials of $-20 \mathrm{mV}$ for rebound and $-80 \mathrm{mV}$ for adaptation. An intrinsic, fixed-value (0.001) conductance $g_{\text {leak }}$ with equilibrium potential of $-35 \mathrm{mV}$ was used to simulate spontaneous firing.

Spike threshold varied in the model as observed in experimental data traces; this was implemented by increasing threshold by a fixed amount $\Delta$ thresh ( 0.6 and $0.2 \mathrm{mV}$ for neurons $\mathrm{A}$ and $\mathrm{B}$, respectively) after each action potential (Liu and Wang, 2001). This threshold increase was set to decay exponentially between spikes with a time constant of $100 \mathrm{msec}$. Spike threshold at the beginning of simulations was set to $-55 \mathrm{mV}$, and to $-45 \mathrm{mV}$ in the fixed-threshold models.

The input current $I_{\text {input }}$ was either a rectangular pulse of $1 \mathrm{sec}$ duration or several seconds of a sinusoidally modulated waveform. Evoked firing was simulated by an additional small constant input current.

The values of $\Delta g_{P R F \max }, \Delta g_{F R A}, \tau_{\mathrm{PRF}}, \tau_{\mathrm{FRA}}$, and $\Delta$ thresh were initially adjusted until the model closely approximated real neuronal responses to hyperpolarizing and depolarizing current pulses. No further adjustments were made to describe the response to sinusoidal current injection.

\section{RESULTS}

To investigate intrinsic firing dynamics, we made whole-cell patch recordings from MVN neurons in slices of the mouse brainstem and analyzed firing rate responses to hyperpolarizing, depolarizing, and sinusoidally modulated stimuli presented with intracellular injection of current. MVN neurons varied markedly in their intrinsic firing properties, in particular, in the extent of PRF and FRA. Both PRF and FRA depend on the immediate history of firing and therefore could, in principle, influence the phase of neuronal responses to sinusoidal inputs. We describe each of these properties in turn and then examine the underlying ionic mechanisms.

\section{Postinhibitory rebound firing}

PRF was observed in a subset of MVN neurons after membrane hyperpolarization evoked by either synaptic or intracellular stimuli. Stimulation of inhibitory axons lateral to the MVN with pulse trains produced a pause in firing that lasted for the duration of the stimulus and was followed by a transient increase in firing rate (Fig. 1A). This increase usually lasted for 1-2 sec, during which the firing rate returned to its baseline value along an exponential time course. The effect of trains of IPSPs could be mimicked by applying hyperpolarizing current pulses through the recording electrode (Fig. 1B). Examples of a neuron with PRF and one that lacked PRF are shown in Figure $1, B$ and $C$, respectively.

To compare PRF across MVN neurons, each neuron was examined at a firing rate of $\sim 10$ spikes/sec, corresponding to the typical resting discharge in vitro; silent neurons were slightly depolarized by DC current injection to fire in this range. A standardized value for PRF was taken as the peak PRF after a 1 sec hyperpolarizing current pulse which lowered the average membrane potential by $\sim 30 \mathrm{mV}$. The peak changes in firing rate observed ranged from -8 to $>100$ spikes/sec. In an unbiased sample (the first 70 neurons recorded for this study), we found 51 neurons with PRF of 10 spikes/sec or less, and only six with rebound of $>20$ spikes/sec.

\section{Properties of neurons with postinhibitory rebound firing}

Figure $2 A$ plots the input resistance of $132 \mathrm{MVN}$ neurons as a function of their peak PRF. This distribution formed a continuum, but it was clear that neurons with low and high PRF differed substantially. To facilitate the analysis we grouped the neurons with lowest PRF and the highest PRF. Three populations were thus designated: neurons with weak ( $\leq 10$ spikes/sec), medium (11-30 spikes/sec), and strong (>30 spikes/sec) PRF. A comparison of several physiological and morphological parameters across these groups is presented in Table 1. Neurons with medium or strong rebound firing had significantly lower input resistances than those that displayed weak PRF $(p<0.001)$. Because input resistance tends to decrease with cell surface area, this difference likely distinguished smaller from larger neurons in our sample. Indeed, when a fluorescent dye was included in the recording solution, neurons with strong PRF were found to have the largest somata and possess a multipolar morphology (Table 1). Neurons with weak PRF, on the other hand, tended to be of small size and displayed fewer proximal processes. Of 18 filled neurons, two are shown in Figure $2 B$. Approximate cell size was also estimated from 96 DIC images acquired during whole-cell recordings (Ta- 


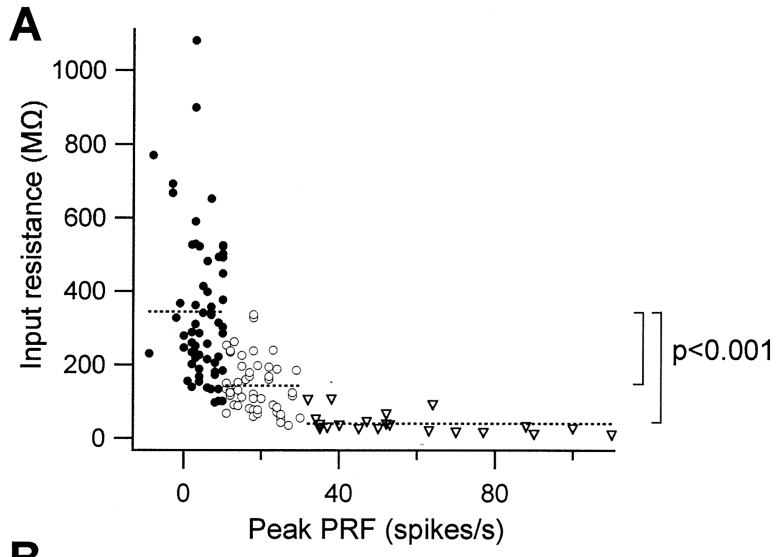

B

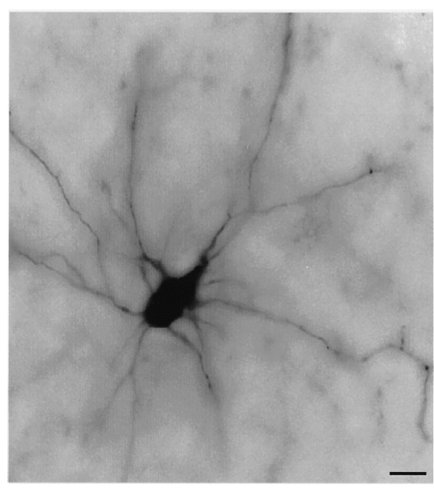

PRF = 77 spikes $/ \mathrm{s}$

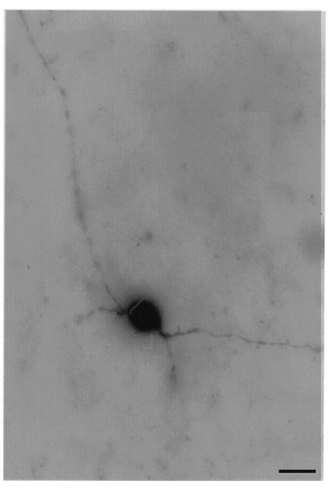

PRF = 5 spikes $/ \mathrm{s}$
Figure 2. Properties of neurons with postinhibitory rebound firing. $A$, Input resistance depends on peak PRF. Dashed lines separate 132 neurons into groups with weak PRF (filled circles), medium PRF (open circles), and strong PRF (triangles). Neurons with high input resistance displayed less rebound firing. $B$, Example of a neuron with strong PRF (left) and one with weak rebound firing (right). Neurons were filled with tetramethylrhodamine dextran. Scale bars, $25 \mu \mathrm{m}$.

ble 1). Neurons with strong PRF had significantly larger areas than those with weak or medium PRF $(p<0.001)$. Taken together, these results demonstrate that larger neurons displayed the strongest rebound firing.

Spike shape also differed between neurons with weak or strong rebound firing (Table 1). Neurons with little PRF had significantly wider action potentials than did those in the medium and strong PRF group $(p<0.001)$. Weak PRF neurons also had significantly larger AHPs than both other groups $(p<0.001)$. Both action potential width and AHP magnitude varied continuously across neurons in our sample, and histograms of these two parameters were well described by a unimodal Gaussian distribution. On the basis of these parameters, neurons in our sample with weak PRF would be classified as type A, whereas those with medium to strong PRF would be classified as type B (Serafin et al., 1991a; Johnston et al., 1994). The slope of the $f-I$ curve (firing response gain) was significantly lower in strong PRF neurons compared with both other groups $(p<0.005)$ (Table 1$)$, indicating that stronger PRF was not caused by higher neuronal excitability. In summary, MVN neurons differed considerably in their firing response after hyperpolarizations, and strong PRF was associated with low input resistance, low firing response gain, and a large multipolar morphology.

\section{Postinhibitory rebound firing depends on inhibition magnitude and duration}

To determine the extent to which PRF can be elicited by synaptic inputs, we examined responses to trains of IPSPs evoked by stimulating inhibitory axons at frequencies between 10 and $70 \mathrm{~Hz}$ (Fig. 1 $A, 3 A$ ). Although the stimulating electrode was in the region of Purkinje cell axons, the identity of the inhibitory inputs stimulated could not be unambiguously determined. Membrane hyperpolarization increased with stimulation frequency such that hyperpolarizations of $>10 \mathrm{mV}$ could be produced with $60-80 \mathrm{~Hz}$ trains of IPSPs. Peak PRF increased linearly with the stimulation frequency (Fig. 3A, bottom), as observed in each of six neurons tested [average $R^{2}=0.91 \pm 0.03$ with slopes of $0.10 \pm 0.02$ (spikes/sec)/Hz]. Therefore, the faster inhibitory synaptic inputs fire, the stronger the evoked rebound firing in MVN neurons.

To extend the range of membrane hyperpolarizations beyond that evokable by electrical stimulation of synaptic inputs, responses to steps of intracellularly injected current were analyzed. PRF depended strongly on membrane hyperpolarization. The top panel in Figure $3 B$ shows firing responses of a single MVN neuron to two different amplitude currents steps. As evidenced by the bottom panel of Figure $3 B$, PRF increased with membrane hyperpolarization. Similar results were obtained in each of 16 neurons tested.

The duration of membrane hyperpolarization also influenced PRF. As shown in the example neuron at the top of Figure $3 C$, current steps lasting $25 \mathrm{msec}$ evoked little PRF, whereas steps of $2 \mathrm{sec}$ evoked strong PRF. The summary plot in Figure $3 C$ (bottom) shows averages across all six neurons tested in this manner, normalized to the maximum value of PRF. The relationship between PRF and pulse duration was well described as a monoexponential process with a time constant of $\sim 600 \mathrm{msec}$.

\section{Firing rate adaptation}

MVN neurons responded to depolarizing current injection with an increase in firing rate, which gradually declined (adapted) during the duration of the step. Figure $4 A$ shows examples of firing rate responses to families of current steps in two example neurons. The neuron in the right panel adapted significantly more than did the neuron in the left panel. To quantify these results, two measures of FRA were calculated (see Materials and Methods): the change in firing rate during the current step (absolute adaptation), and the ratio of the firing rate at the end and the rate near the beginning of the step (adaptation ratio). The neurons in Figure $4 A$ had absolute adaptation values of 0.5 and 24 spikes/sec, and their adaptation ratios were 0.98 and 0.54 , respectively.

The adaptation ratio ranged from 0.36 to 1.06 across our sample of MVN neurons $(n=58)$ and was correlated with the magnitude of postinhibitory rebound firing. Figure $4 B$ plots the adaptation ratio as a function of peak PRF in 66 neurons; the correlation coefficient $\left(R^{2}\right)$ was 0.70 , indicating that pronounced rebound firing was typically accompanied by strong adaptation.

The dependence of FRA on firing rate differed across neurons with strong and weak adaptation. In the examples shown in Figure $4 A$, the weakly adapting neuron exhibited substantial adaptation only at high firing rates (responses to large current steps), whereas the strongly adapting neuron showed considerable FRA at all firing rates. Figure $4 C$ shows population data divided into two groups of neurons with low and high PRF (11-19 spikes/sec and $>50$ spikes/sec, respectively). Neurons in the low PRF (and low FRA) group showed an increase in absolute adaptation (filled circles) with firing rate, whereas the adaptation 
Table 1. Physiological and morphological characterization of MVN neurons

\begin{tabular}{|c|c|c|c|c|c|c|}
\hline & $\begin{array}{l}R_{\mathrm{in}} \\
(M \Omega)\end{array}$ & $\begin{array}{l}\text { Gain } \\
\left(\text { spikes/sec } \cdot \mathrm{nA}^{-1}\right)\end{array}$ & $\begin{array}{l}\text { Spike width } \\
\text { (msec) }\end{array}$ & $\begin{array}{l}\text { AHP } \\
(\mathrm{mV})\end{array}$ & $\begin{array}{l}\text { DIC area } \\
\left(\mu \mathrm{m}^{2}\right)\end{array}$ & $\begin{array}{l}\text { Primary } \\
\text { processes }\end{array}$ \\
\hline $\begin{array}{l}\text { Weak PRF } \\
(\text { PRF } \leq 10 \text { spikes/sec })\end{array}$ & $\begin{aligned} 345 & \pm 25 \\
(n & =62)\end{aligned}$ & $\begin{aligned} 182 & \pm 11 \\
(n & =53)\end{aligned}$ & $\begin{array}{c}1.04 \pm 0.04 \\
(n=76)\end{array}$ & $\begin{aligned} 26.2 & \pm 0.6 \\
(n & =76)\end{aligned}$ & $\begin{aligned} 168 & \pm 9 \\
(n & =48)\end{aligned}$ & $\begin{aligned} 2.5 & \pm 0.4 \\
(n & =6)\end{aligned}$ \\
\hline $\begin{array}{l}\text { Medium PRF } \\
\text { (PRF 11-30 spikes/sec) }\end{array}$ & $\begin{aligned} 144 & \pm 10 \\
(n & =49)\end{aligned}$ & $\begin{aligned} 149 & \pm 6 \\
(n & =40)\end{aligned}$ & $\begin{aligned} 0.82 & \pm 0.03 \\
(n & =49)\end{aligned}$ & $\begin{aligned} 20.9 & \pm 0.5 \\
(n & =49)\end{aligned}$ & $\begin{aligned} 225 & \pm 16 \\
(n & =34)\end{aligned}$ & $\begin{array}{c}4.3 \pm 0.3 \\
(n=7)\end{array}$ \\
\hline $\begin{array}{l}\text { Strong PRF } \\
(\mathrm{PRF}>30 \text { spikes/sec })\end{array}$ & $\begin{array}{l}40 \pm 6 \\
(n=21)\end{array}$ & $\begin{array}{l}88 \pm 7 \\
(n=19)\end{array}$ & $\begin{aligned} 0.69 & \pm 0.04 \\
(n & =21)\end{aligned}$ & $\begin{array}{r}17.7 \pm 0.6 \\
(n=21)\end{array}$ & $\begin{aligned} 383 & \pm 41 \\
(n & =14)\end{aligned}$ & $\begin{array}{c}7.2 \pm 0.8 \\
(n=5)\end{array}$ \\
\hline
\end{tabular}



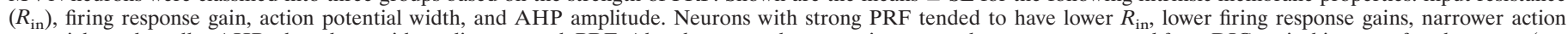

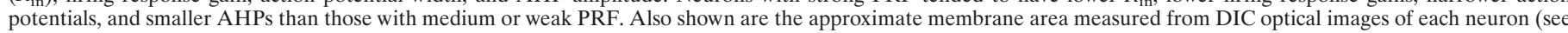

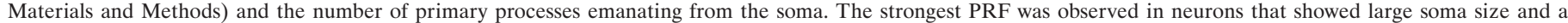
multipolar morphology.
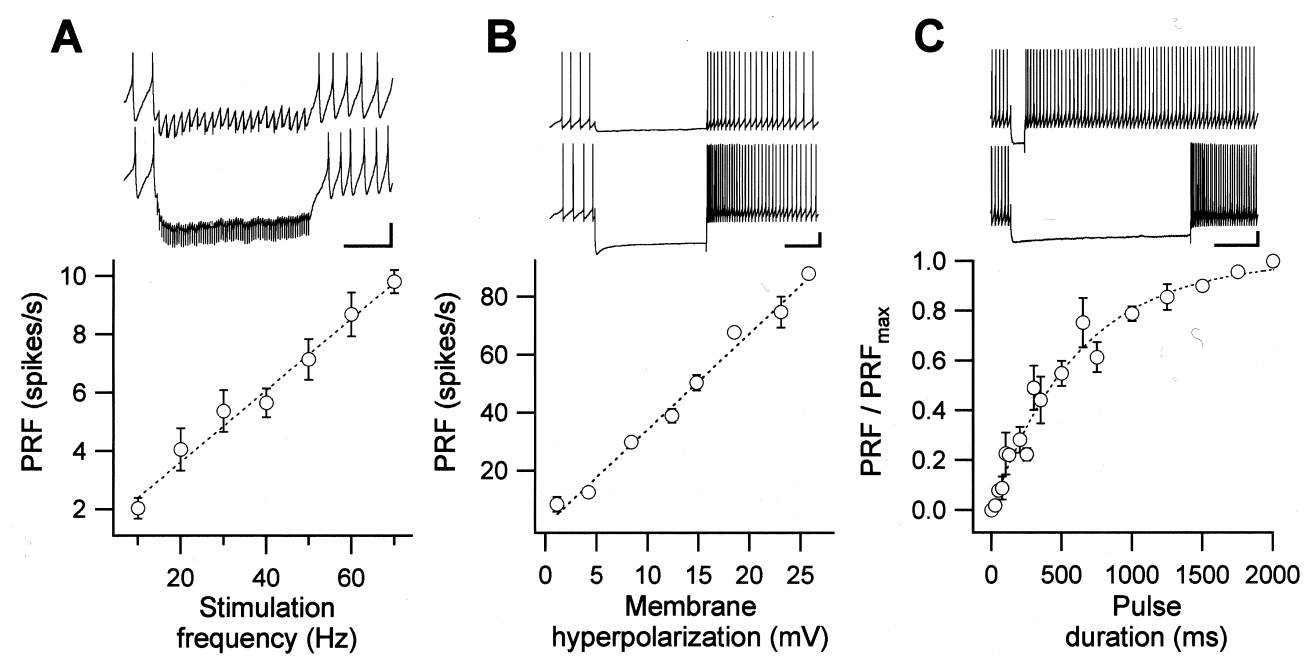

Figure 3. PRF magnitude depends on amplitude and duration of inhibition. $A$, Effects of inhibitory synaptic stimulus frequency on PRF. Top traces, Examples of membrane potential responses to inhibitory synaptic stimulation at 20 and $70 \mathrm{~Hz}$ in a single neuron (resulting average hyperpolarizations were -4 and $-10 \mathrm{mV}$ for the 20 and $70 \mathrm{~Hz}$ trains, respectively). Action potentials are truncated. Calibration: $5 \mathrm{mV}, 300 \mathrm{msec}$. Bottom traces, Average evoked rebound firing is plotted against synaptic stimulation frequency in the same neuron. Error bars are SE of the peak firing rate, evoked by three repetitions at each frequency. $B$, Effects of membrane hyperpolarization on PRF. Top traces, Examples of membrane potential traces in response to two different current step injections $(-150$ and $-1200 \mathrm{pA})$. Calibration: $10 \mathrm{mV}$ and $300 \mathrm{msec}$. Bottom traces, Average rebound firing, plotted as a function of membrane hyperpolarization during the current step in the same neuron. Error bars represent SE of the peak firing rate across three repetitions of each current pulse. $C$, Effects of pulse duration. Top traces, Examples of membrane potential traces in response to current step injections with two different durations (100 and $1300 \mathrm{msec}$ ). Calibration: $10 \mathrm{mV}$, $300 \mathrm{msec}$. Bottom traces, Average rebound response from six neurons, normalized to the maximum PRF in each neuron and plotted as a function of the current pulse duration. Dashed line is an exponential fit with $\tau=620 \mathrm{msec}$.

ratio (crosses) did not depend on firing rate. This signifies that no matter how fast these neurons fired, the response at the end of the step was always proportional to the initial response. Neurons with high PRF (and high FRA), on the other hand, tended to adapt disproportionally less when active at high rates (Fig. 4C, right).

\section{Dynamics of neuronal responses to temporally modulated inputs}

The previous experiments established that in a subset of MVN neurons, sustained membrane hyperpolarization is followed by rebound firing and that those neurons respond to depolarization with strong adaptation of their firing rate. However, input signals to MVN neurons in the behaving animal vary with time, such that hyperpolarizing and depolarizing drive changes significantly during head movements. To better relate intrinsic firing dynamics with response properties measured in behaving animals, we analyzed firing rate responses to sinusoidally modulated input currents.

As shown in Figure $5 A$, sinusoidally alternating depolarization and hyperpolarization (solid line) evoked alternating increases and decreases in firing rate (data points). We analyzed responses to sinusoidal stimuli presented at frequencies between 0.125 and $4 \mathrm{~Hz}$. Neurons often fell silent during the hyperpolarizing phase of large-amplitude stimuli (Fig. $5 B$ ), as can occur during head movements in intact animals (Fuchs and Kimm, 1975). Analysis of traces with or without a cutoff revealed no differences in the resulting phase values (data not shown).

Neurons with strong PRF respond to sinusoidal inputs with a phase lead relative to those with weak PRF. Figure 5, $A$ and $B$, shows examples from two neurons with strong and weak PRF (52 and 2 spikes/sec, respectively). To facilitate the comparisons, the firing rates of the neurons were adjusted to comparable levels with DC current. Responses to sinusoidal stimuli presented at 0.125 and $0.25 \mathrm{~Hz}$ are shown in Figure 5, $A$ and $B$, respectively. In both cases, the response of the neuron with strong PRF ( filled symbols) precedes that of the low-rebound neuron (open symbols). This positive shift along the time axis is referred to as phase lead and was found at all frequencies tested.

To quantify these phase shifts, sinusoidal best-fit curves to the firing rate response were calculated at each frequency and the phase relationship to the input stimulus determined. Sine waves generally fit the data well, with correlation coefficients ranging from 0.95 to 0.99 . For comparison, the $R^{2}$ values for the two neurons shown in Figure 5, $A$ and $B$, were from 0.97 to 0.98 . Figure $5 C$ summarizes the frequency dependence of the phase of groups of neurons with weak and strong PRF, respectively. Firing 
A

A PRF $=11$ spikes $/ \mathrm{s}$

$\mathrm{PRF}=88$ spikes $/ \mathrm{s}$
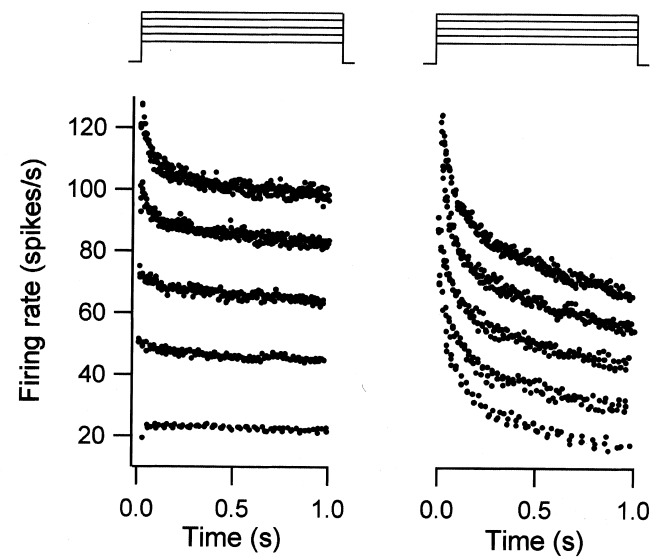

B

B

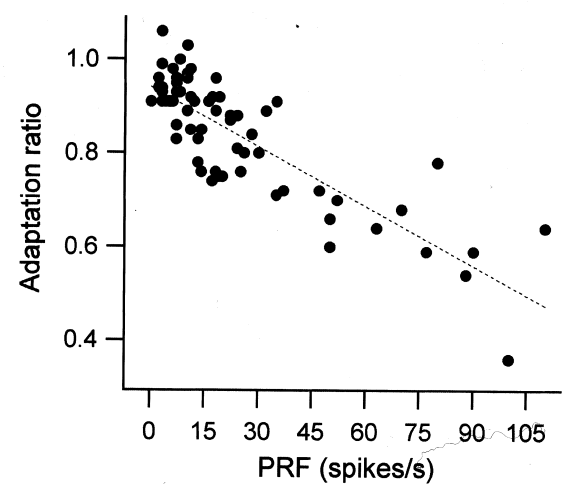

C

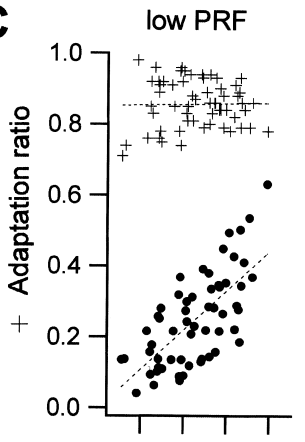

255075100

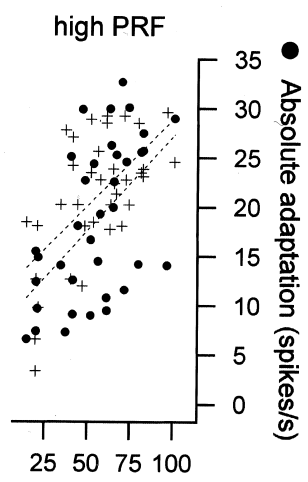

firing rate at step onset (spikes/s)

Figure 4. Firing rate adaptation varies across MVN neurons. $A$, Time course of firing rate changes in response to $1 \mathrm{sec}$ depolarizing current steps in a neuron with PRF of 11 spikes/sec (left) and one with 88 spikes/sec (right). Responses to three repetitions at each current amplitude are shown. Current traces are plotted above. $B$, Summary plot of one measure of FRA (adaptation ratio, see Materials and Methods) as a function of PRF in 66 neurons. Stronger adaptation was seen in neurons with strong PRF. The linear correlation coefficient $\left(R^{2}\right.$; dashed line $)$ was 0.70. $C$, Dependence of FRA on firing rate in neurons similar to the examples shown in $A$. Neurons with PRF of 11-19 spikes/sec (low PRF) showed an increase of adaptation with firing rate (circles; $R^{2}=0.48$ ), but no change in adaptation ratio (crosses; $R^{2}=0.0005 ; n=16$ ). In 10 neurons with high PRF $(>50$ spikes/sec) both measures of FR A increased with firing rate $\left(R^{2}=0.37\right.$ for absolute adaptation; $R^{2}=0.30$ for adaptation ratio).

rate was modulated by approximately $\pm 20-30$ spikes/sec around a baseline of 30 spikes/sec. The first group had an average PRF of $5 \pm 1$ spikes/sec $(n=8)$ and is plotted as open circles in Figure $5 C$. PRF in the second group of neurons averaged $60 \pm 7$ spikes/sec $(n=12)$, shown as filled squares. The firing rate phase advance of the neurons with strong PRF relative to the weak PRF neurons ranged from 10 to $15^{\circ}$ over the range of stimulus frequencies tested. This phase difference between the two groups was significant at all frequencies $(p<0.01)$.

The amount of phase shift in each neuron was also correlated with the amount of firing rate adaptation. Figure $5 D$ exemplifies this for 25 neurons tested at an input frequency of $0.25 \mathrm{~Hz}$. Phase is plotted as a function of adaptation ratio. Larger phase leads were clearly associated with stronger FRA $\left(R^{2}=0.70\right)$. These data indicate that in MVN neurons, the processing of temporally modulated input signals differs considerably between neurons with strong and weak FRA and PRF.

\section{Ionic mechanisms of rebound firing and adaptation}

What ionic currents confer variations in response phase onto MVN neurons? We first examined the mechanisms of rebound firing and adaptation using pharmacological manipulations and then incorporated the results into a simple model to gain insights into mechanisms that underlie the differences in response phase described above.

\section{The hyperpolarization-activated current $\mathrm{I}_{H}$ contributes to rebound firing}

The conductances underlying PRF must fulfill the following criteria, derived from the results presented above: (1) they must be activated by membrane hyperpolarizations in a membrane potential-dependent manner, (2) they must provide a net inward current after the offset of hyperpolarization, and (3) their effects on membrane potential should decay exponentially with a time constant of hundreds of milliseconds. The conductance that mediates the hyperpolarization-activated $\mathrm{H}$ current $\left(I_{\mathrm{H}}\right)$ meets each of these criteria.

Subthreshold activation of $I_{\mathrm{H}}$ produces a transient depolarization after offset of membrane hyperpolarization that can be blocked by low concentrations of $\mathrm{Cs}^{+}$(Pape, 1996). We tested the effect of $I_{\mathrm{H}}$ blockers on $26 \mathrm{MVN}$ neurons and found a range of responses from no change in PRF to a reduction of $>70 \%$. The left column of Figure $6 \mathrm{~A}$ shows an example of a neuron in which $\mathrm{Cs}^{+}(3 \mathrm{~mm})$ lowered PRF substantially. Note that the membrane potential sag (arrow) is absent in the bottom trace in Figure $6 \mathrm{~A}$, indicating a complete block of $I_{\mathrm{H}}$, as observed in all neurons tested. The current amplitudes for the two traces shown were chosen to give the same average membrane hyperpolarization during the step, because PRF depends on hyperpolarization amplitude (Fig. 3B). Data from a different neuron with similar magnitude of PRF are shown in the right column of Figure $6 \mathrm{~A}$. The firing pattern is almost unchanged despite the absence of a sag. Figure $6 B$ shows the firing rate traces corresponding to the neurons in $A$. In the first neuron, rebound firing was reduced by $\sim 80 \%$ in the presence of $\mathrm{Cs}^{+}$; this reduction was evident at all magnitudes of membrane hyperpolarization. In the second neuron, the application of $\mathrm{Cs}^{+}$reduced the peak PRF by only $\sim 20 \%$. Wash-out of $\mathrm{Cs}^{+}$partially recovered the membrane sag and restored PRF (data not shown).

The effect of blocking $I_{\mathrm{H}}$ on PRF is summarized in Figure $6 C$, which plots PRF in the presence of $I_{\mathrm{H}}$ blockers against PRF in control bath medium for 26 neurons. To obtain independent 


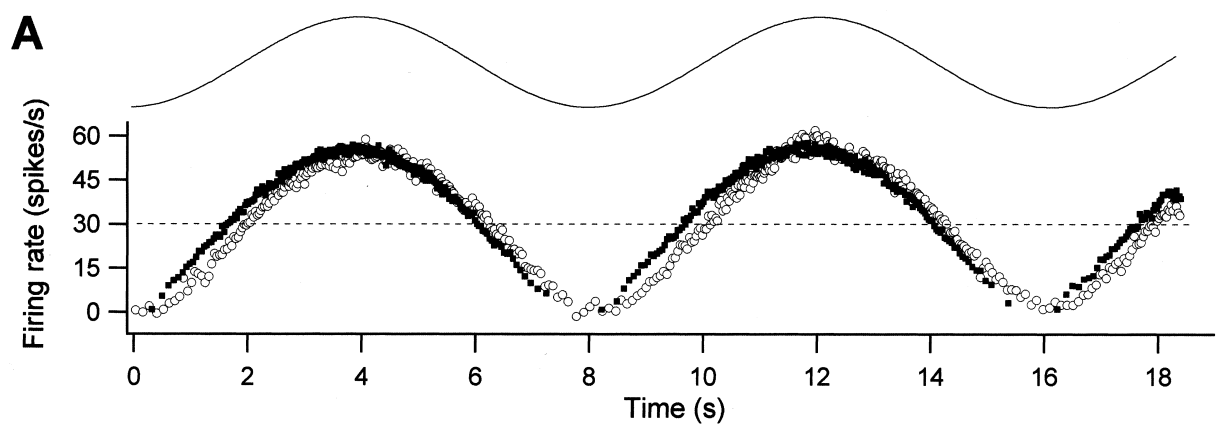

strong PRF neuron (52 spikes/s)

weak PRF neuron (2 spikes/s)
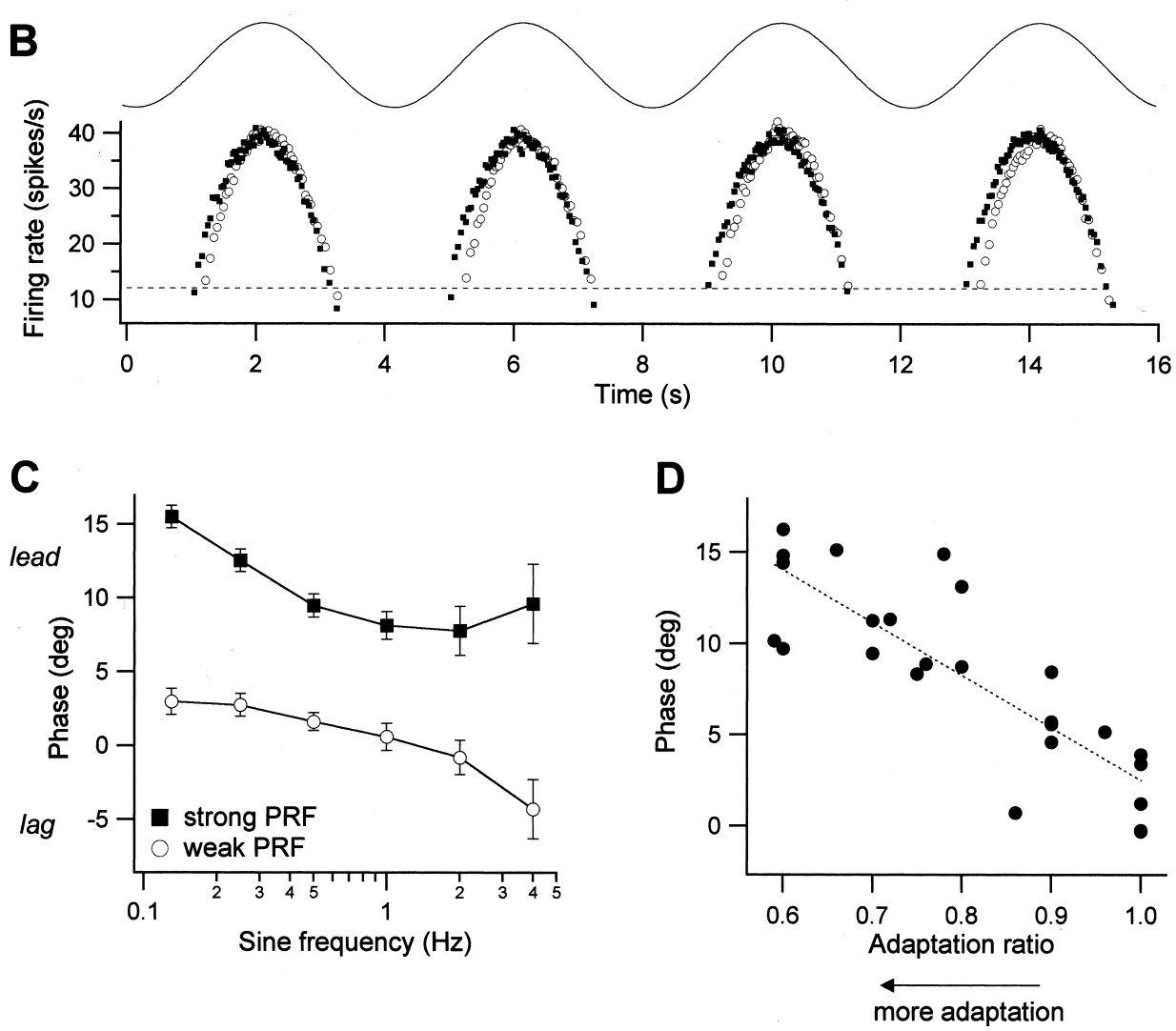

Figure 5. Neurons with strong PRF and FRA experience a phase-lead during sinusoidal current input. $A$, Comparison of a neuron with strong rebound firing ( filled symbols) and one with weak rebound firing (open symbols) as they modulate their firing rate in response to sinusoidal input current at $0.125 \mathrm{~Hz}$. Top trace, Input current versus time; bottom trace, firing rate versus time. The phase of the neuron with strong PRF was $+16^{\circ}$, and the phase of the neuron with weak PRF was $+4^{\circ}$, relative to the input sine wave. The difference between the two neurons can be clearly seen as a relative offset along the time axis. Dashed line indicates the baseline firing rate before current injection (30 spikes/sec). $B$, Response of the same neurons shown in $A$ to a $0.25 \mathrm{~Hz}$ stimulus. Baseline firing rate of 12 spikes/sec is indicated by the dashed line. The phases were $+11^{\circ}$ and $+2^{\circ}$ for the strong and the weak PRF neuron, respectively. $C$, The phase of best-fitting sine waves is plotted against input frequency. Shown are averages for 12 neurons with strong PRF (>30 spikes/sec, mean $60 \pm 7$ spikes/sec; filled symbols), and eight neurons with weak PRF ( $\leq 10$ spikes/sec, mean $5 \pm 1$ spikes/sec; open symbols). Data from firing rate modulation around 30 spikes/sec (as in $A$ ) were used to generate this plot. $D$, Summary plot of the phase of 25 neurons as a function of adaptation. Phase was measured at an input frequency of $0.25 \mathrm{~Hz}$ and was well correlated with FRA (dashed line; $R^{2}=$ $0.70)$. verification of the results with $\mathrm{Cs}^{+}$, the selective $I_{\mathrm{H}}$ blocker ZD7288 was substituted for $\mathrm{Cs}^{+}$in seven neurons ( filled circles); no differences were seen between the two pharmacological agents. The dashed line in Figure $6 C$ denotes the case of $I_{\mathrm{H}}$ having no effect on PRF. The data lie below this line in all but one neuron tested, indicating that $I_{\mathrm{H}}$ contributes to PRF, although this contribution varies substantially across neurons. Note that there is an apparent plateau of PRF in $I_{\mathrm{H}}$ blockers, indicating that a residual, $I_{\mathrm{H}^{-}}$-independent component contributes a maximum of $\sim 30$ spikes/sec to PRF.

The contribution of $I_{\mathrm{H}}$ to PRF depended on baseline firing rate. In five neurons firing at $10 \pm 1$ spikes/sec, blocking $I_{\mathrm{H}}$ reduced PRF by $50 \pm 7 \%$ (data not shown). However, when the same neurons were depolarized to fire at $30 \pm 0.5 \mathrm{spikes} / \mathrm{sec}, I_{\mathrm{H}}$ blockade reduced PRF by only $26 \pm 8 \%$, indicating that given the same input current, $I_{\mathrm{H}}$ contributes more strongly to PRF when neurons fire slowly and that an $I_{\mathrm{H}}$-independent mechanism dominates PRF at higher baseline firing rates.
Blocking $I_{\mathrm{H}}$ did not alter firing rate adaptation. Figure $6 D$ shows the firing rate responses to depolarizing current steps in a neuron with strong PRF. Although $\mathrm{Cs}^{+}$reduced PRF by $34 \%$ (data not shown), the magnitude and time course of FRA was unaffected. In 12 neurons tested, the average adaptation ratio before and after application of $I_{\mathrm{H}}$ blockers was $0.83 \pm 0.03$ and $0.83 \pm 0.04$, respectively. Consistent with these findings, $I_{\mathrm{H}}$ blockers did not induce a significant change in firing response gain (gain in $\mathrm{Cs}^{+}$or ZD7288 was $103 \pm 4 \%$ of control; $n=12$ ).

The phase lead observed in strong PRF neurons (Fig. 5) was not affected by blocking $I_{\mathrm{H}}$. Figure $6 E$ shows average phase in response to sinusoidal current injection before and after $I_{\mathrm{H}}$ blocker application. Care was taken to avoid cutoff during the trough of the sine waves to eliminate confounding subthreshold membrane potential changes because of increases in input resistance induced by $I_{\mathrm{H}}$ blockers. No change in the phase of the firing response was evident, despite an average of $43 \%$ reduction of $\operatorname{PRF}(n=3)$. These data suggest that it is unlikely that variations 
A

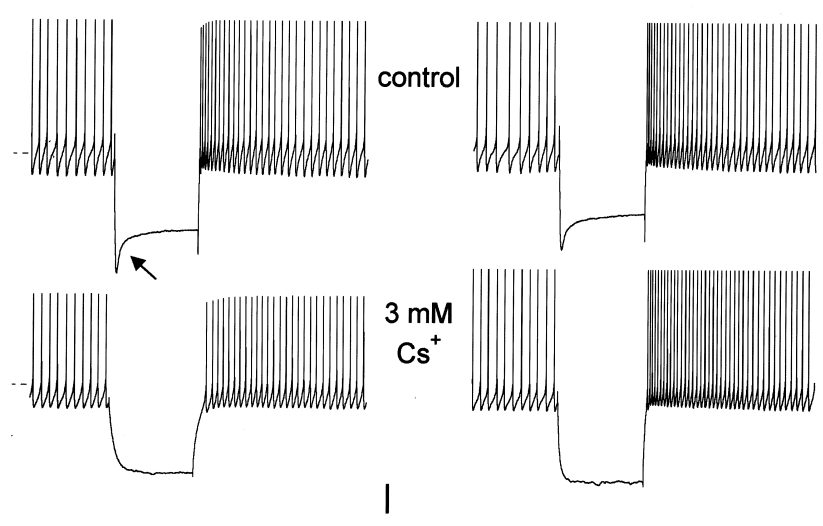

C

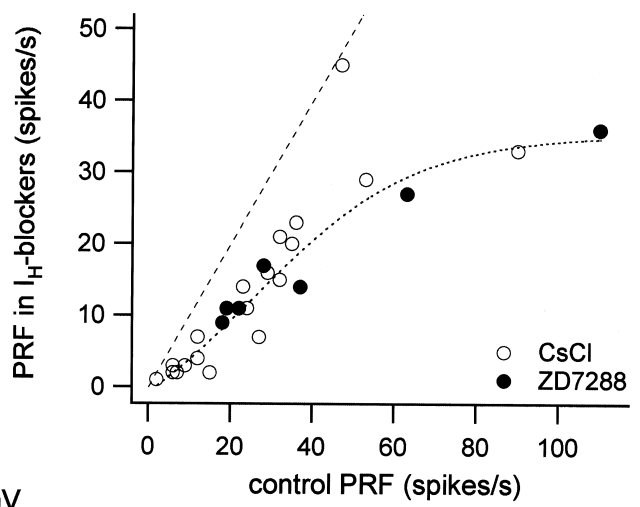

B

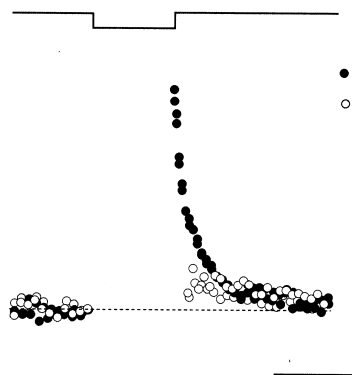

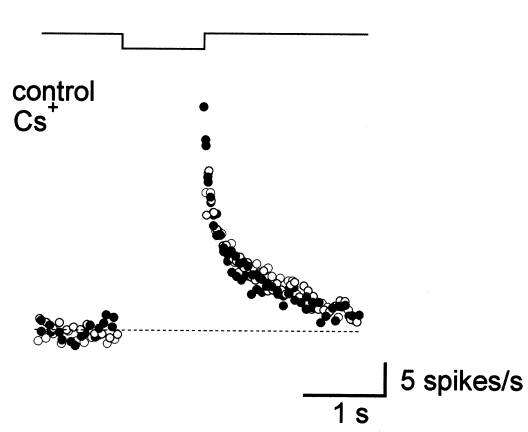

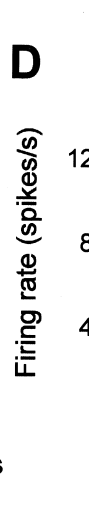

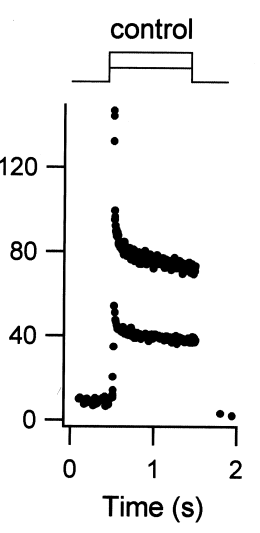

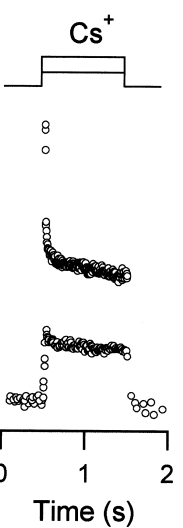

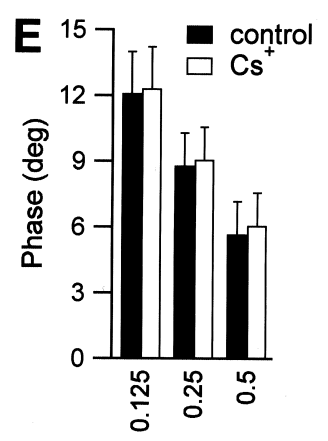

Sine frequency $(\mathrm{Hz})$

Figure 6. The hyperpolarization-activated current $I_{\mathrm{H}}$ contributes to PRF, but not to FRA or phase. $A$, Responses to hyperpolarizing current steps in two MVN neurons. Left column, Example of a neuron with PRF dominated by $I_{\mathrm{H}}$. Membrane potential traces as a function of time for control (top trace) and with bath-applied $3 \mathrm{~mm} \mathrm{Cs}^{+}$, a blocker of $I_{\mathrm{H}}$ (bottom trace). Note that the membrane sag seen in the control trace (arrow) was abolished in the presence of $\mathrm{Cs}^{+}$. The injected current amplitudes were adjusted so that the average hyperpolarizations produced by both current steps were matched. Right column, Example of a neuron in which $I_{\mathrm{H}}$ contributed little to rebound firing. Dashed lines indicate $-65 \mathrm{mV}$. B, Instantaneous firing rates versus time for the traces shown in $A$, before ( filled circles) and after $\mathrm{Cs}^{+}$application (open circles). Baseline firing rates were $\sim 10$ spikes/sec. $C$, Summary plot of PRF before versus after the application of one of two $I_{\mathrm{H}}$ blockers (3-5 mM Cs ${ }^{+}$or 30-100 $\mu \mathrm{M} \mathrm{ZD7288)}$ in 26 neurons. The dashed line indicates the case of no effect of blocking $I_{\mathrm{H}}$, and the dotted line is a sigmoidal fit to the data. $D$, Time course of firing rate responses to depolarizing current steps before and after the application of $\mathrm{Cs}^{+}(5 \mathrm{~mm})$ in a neuron with PRF of 32 spikes/sec. In this neuron, $\mathrm{Cs}^{+}$reduced PRF to $21 \mathrm{spikes} / \mathrm{sec}$. FRA, however, was not affected by blocking $I_{\mathrm{H}}$ (adaptation ratios were 0.89 and 0.91 in control and Cs ${ }^{+}$bath solution, respectively). $E$, Effect of $I_{\mathrm{H}}$ blockers on response phase during sinusoidal current injections at three frequencies. Control, Black bars; $\mathrm{Cs}^{+}(3 \mathrm{~mm})$, white bars. Data are means \pm SE for three neurons.

in PRF contribute significantly to phase differences in MVN neurons.

In summary, although rebound firing, adaptation, and response phase are correlated in MVN neurons (Figs. $4 B, 5 D$ ), these measures of firing response dynamics are mediated by ionic currents that differentially depend on $I_{\mathrm{H}}$. These results raise the possibility that an $I_{\mathrm{H}^{-}}$-independent component of PRF may underlie both FRA and phase lead.

Rebound can be fully eliminated by blocking $\mathrm{I}_{H}$ and $\mathrm{I}_{N a}$ What current or currents mediate the residual PRF observed when $I_{\mathrm{H}}$ is blocked? In many cell types, $\mathrm{Ca}^{2+}$ currents contribute to low-threshold spikes after the offset of membrane hyperpolarization (Huguenard, 1996). We investigated whether $\mathrm{Ca}^{2+}$ currents contributed to PRF in MVN neurons by first blocking $I_{\mathrm{H}}$ and then reducing $\mathrm{Ca}^{2+}$ influx through voltage-sensitive $\mathrm{Ca}^{2+}$ channels by either removing $\mathrm{Ca}^{2+}$ from the extracellular bath or applying the $\mathrm{Ca}^{2+}$ channel blocker cadmium $(100 \mu \mathrm{M})$. As expected, $I_{\mathrm{H}}$ blockade reduced PRF (by $40 \pm 4 \%$; $n=3$ ); however, subsequent reduction of calcium influx evoked an increase in PRF (by $323 \pm 49 \% ; n=3$ ), indicating that in these neurons, $\mathrm{Ca}^{2+}$ currents do not mediate the $I_{\mathrm{H}}$-independent component of PRF. The increase in PRF would be expected given that in MVN neurons, reduction of $\mathrm{Ca}^{2+}$ influx leads to increases in excitability (Smith et al., 2002) and thereby would result in an enhancement of firing resulting from residual rebound currents.

To further investigate these residual currents, we blocked voltage-dependent $\mathrm{Na}^{+}$channels with $1 \mu \mathrm{M}$ tetrodotoxin (TTX). Figure 7 shows a neuron with strong PRF (63 spikes/sec; left trace) in which PRF was reduced, but not eliminated, after application of ZD7288 (middle trace). The addition of TTX blocked firing but failed to reveal a rebound depolarization that could underlie PRF (right trace). A depolarizing "hump" would be expected if the rebound spikes merely rode on top of a membrane depolarization (Jahnsen and Llinas, 1984; Serafin et al., 1991b).

A combination of TTX together with $\mathrm{Cs}^{+}$or ZD7288 completely eliminated postinhibitory rebound in 12 neurons of 16 tested. The remaining four neurons displayed small residual rebound depolarizations in the presence of both drugs, which in two neurons were eliminated by blocking $\mathrm{Ca}^{2+}$ channels with cadmium (100 $\mu \mathrm{M}$; data not shown), indicating that a small percent- 

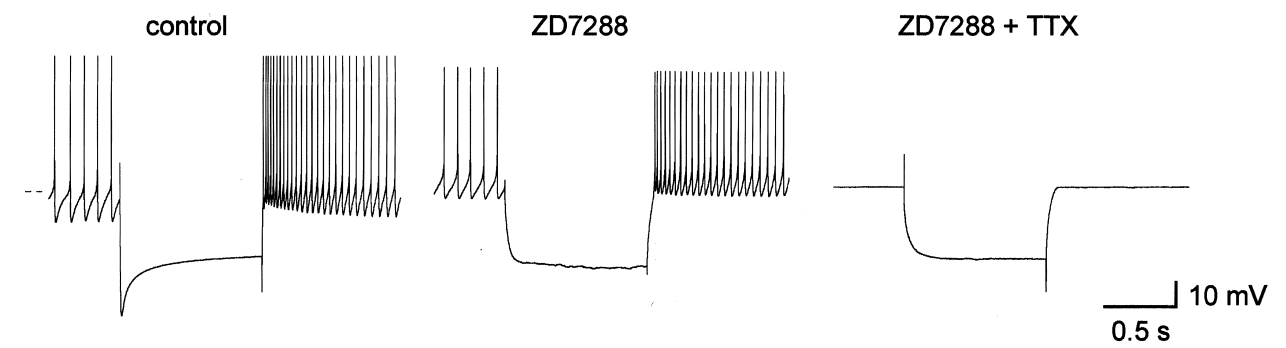

Figure 7. Rebound can be completely blocked by a combination of $I_{\mathrm{H}}$ and $I_{\mathrm{Na}}$ antagonists. Membrane potential traces are plotted versus time under three different conditions in a single neuron. After eliminating the $I_{\mathrm{H}}$ component with $100 \mu \mathrm{M}$ ZD7288, PRF in this neuron was reduced from 63 (left trace) to 27 spikes/sec (center trace). Addition of the Na channel blocker TTX eliminated all postinhibitory rebound depolarization (right trace), indicat-

ing that either spiking or a TTX-sensitive $\mathrm{Na}^{+}$current is necessary for the $I_{\mathrm{H}^{-}}$-insensitive component of PRF. Dashed line indicates $-60 \mathrm{mV}$.

age of MVN neurons have a calcium contribution to rebound, most likely mediated by low-threshold $\mathrm{Ca}^{2+}$ channels (Serafin et al., 1990, 1991b; Huguenard, 1996; Aizenman and Linden, 1999).

The $I_{\mathrm{H}^{-}}$and $\mathrm{Ca}^{2+}$-independent component of PRF could either be mediated by a TTX-sensitive sodium current or by other currents that are activated during action potentials. We found little evidence for a subthreshold $\mathrm{Na}^{+}$current evoked by our PRF protocol. Such a current would be expected to evoke a TTXsensitive depolarizing responses while the membrane is hyperpolarized (Jahnsen and Llinas, 1984). However, after blockade of $I_{\mathrm{H}}$ with ZD7288, 11 of 12 neurons tested did not exhibit a TTXsensitive depolarization when hyperpolarized below spike threshold. Thus, a better explanation for the mechanisms underlying $I_{\mathrm{H}^{-}}$-independent PRF may be found in currents activated during spiking.

\section{Firing rate adaptation is mediated by $\mathrm{K}^{+}$currents, but is} $\mathrm{Ca}^{2+}$-independent

In many types of neurons, firing rate adaptation is mediated by potassium currents that accumulate with successive action potentials (Baldissera et al., 1978; Sah and Davies, 2000). To investigate the role of $\mathrm{K}^{+}$currents in FRA in MVN neurons, we reduced extracellular $\left[\mathrm{K}^{+}\right]$from 5 to $1 \mathrm{mM}$. This lowered the $\mathrm{K}^{+}$equilibrium potential from -87 to $-130 \mathrm{mV}$. If $\mathrm{K}^{+}$currents contribute to FRA, their effect would be expected to be larger in low $\mathrm{K}^{+}$ because of the resultant increase in driving force on $\mathrm{K}^{+}$ions. As shown in Figure $8 A$, FRA indeed increased in low $\mathrm{K}^{+}$; the average adaptation ratio decreased from $0.87 \pm 0.03$ to $0.72 \pm$ $0.07(n=6 ; p<0.05)$, and the average absolute adaptation more than doubled in low $\mathrm{K}^{+}$solution.

Repetitive firing is often followed by a long-lasting hyperpolarization (slow AHP) that is usually attributed to the accumulation of same current that underlies FRA (Azouz et al., 1996; Sanchez-Vives et al., 2000; Faber et al., 2001). Strongly adapting MVN neurons showed larger slow AHPs than weakly adapting ones $\left(R^{2}=0.59 ; n=19\right)$. Furthermore, the slow AHP was increased in low $\mathrm{K}^{+}$extracellular solution. This is shown in two superimposed membrane potential traces in Figure $8 B$, before and after reduction of $\mathrm{K}^{+}$. The slow AHP was larger when the driving force for $\mathrm{K}^{+}$ions was increased, indicating that a $\mathrm{K}^{+}$ current contributed to this hyperpolarization. Similar increases were found in five of six neurons tested. Moreover, increasing extracellular $\mathrm{K}^{+}$significantly decreased the slow AHP $(n=3$; data not shown). These data are consistent with the hypothesis that slow $\mathrm{K}^{+}$currents underlie adaptation in MVN neurons.

The activation of $\mathrm{K}^{+}$currents by calcium influx during repetitive action potentials mediates firing rate adaptation in many central neurons (Sah and Davies, 2000). To investigate whether $\mathrm{Ca}^{2+}$-dependent $\mathrm{K}^{+}$currents play a role in FRA in MVN neurons, we removed $\mathrm{Ca}^{2+}$ from the extracellular medium, thereby precluding $\mathrm{Ca}^{2+}$ influx during the action potential. Fig- ure $8 C$ shows an example of FRA under control conditions and in a bath solution containing zero $\mathrm{Ca}^{2+}$. Adaptation was not reduced in any of three neurons tested, and was often found to increase in magnitude, as has been observed in cortical neurons (Sanchez-Vives et al., 2000). Similar results were obtained when the $\mathrm{Ca}^{2+}$ channel blocker cadmium was added to the bath or when the $\mathrm{Ca}^{2+}$ chelator BAPTA was included in the internal recording solution (data not shown). These results indicate that $\mathrm{Ca}^{2+}$-dependent $\mathrm{K}^{+}$currents do not mediate FRA. Consistent with these results, the slow AHP was not blocked by removal of extracellular $\mathrm{Ca}^{2+}(n=4$; data not shown).

Together, these results suggest that currents activated during spiking, including $\mathrm{Ca}^{2+}$-independent $\mathrm{K}^{+}$currents, contribute to adaptation.

\section{Firing response dynamics modeled}

A lack of specific pharmacological antagonists to $\mathrm{K}^{+}$currents activated during spiking precluded experimental determination of the relative contributions of the currents underlying PRF and FRA to phase response in MVN neurons. To further explore the mechanisms of response dynamics, we constructed an integrateand-fire model that captured the dynamics of both PRF and FRA in a quantitative manner. This type of model was chosen because it readily produces realistic spiking behavior without requiring detailed knowledge about the properties of every conductance expressed in MVN neurons. It reproduces firing responses of MVN neurons with a small number of variable parameters.

To simulate neuronal responses to hyperpolarizing and depolarizing current pulses, we added two ionic conductances to an integrate-and-fire model: a hyperpolarization-activated conductance and a spike-dependent potassium conductance. The membrane potential dependence of activation and time constants of decay of these conductances approximated those inferred from the results of our pharmacological experiments and from the literature (see Materials and Methods) and were set by matching model responses to depolarizing and hyperpolarizing steps to those of representative MVN neurons. The model also incorporated a variable spike threshold (see Materials and Methods), which did not change the results qualitatively, but was required to reproduce quantitatively the pattern of phase versus frequency, as discussed below. The dependence of PRF magnitude on hyperpolarization amplitude (Fig. 3B) and duration (Fig. 3C) were mimicked by the model (data not shown): PRF increased linearly with current step magnitude and exponentially with step duration. Similarly, adaptation increased linearly with baseline firing rate (as in Fig. 4C).

Figure 9 shows data and model results for two representative neurons with strong FRA and PRF (neuron A) and weak FRA and PRF (neuron B). Figure 9, A1 and B1, shows that the responses to depolarizing steps were reproduced well between 0 and 80 spikes/sec in both neurons. The adaptation conductance 
A

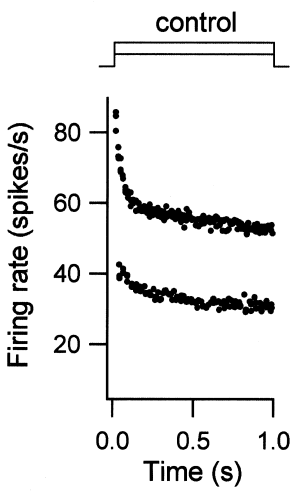

B
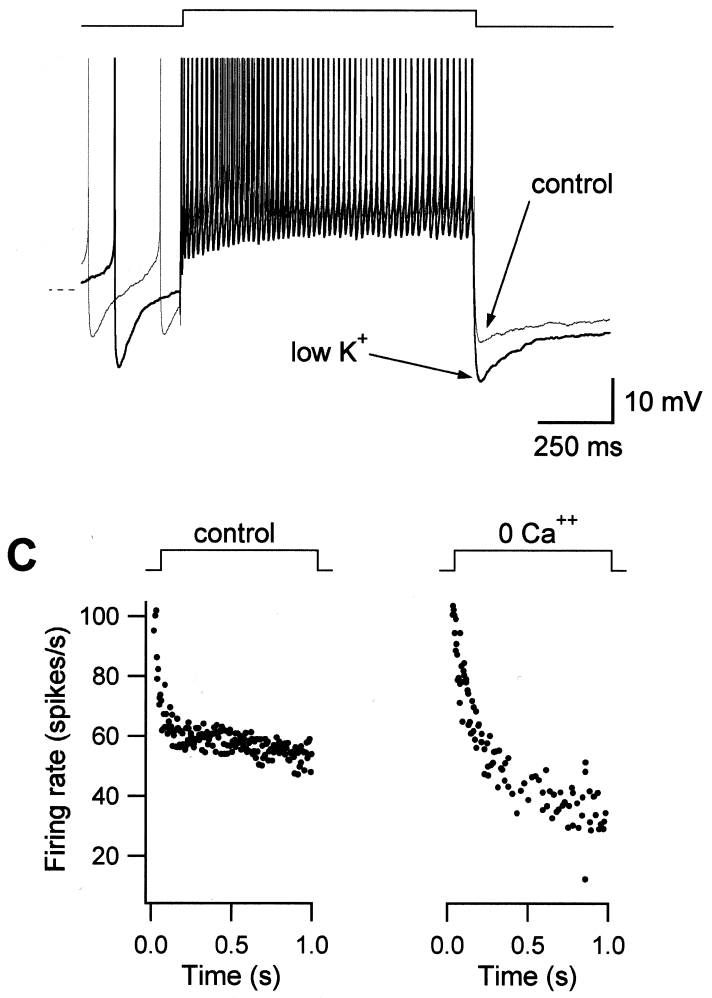

Figure 8. Spike-dependent $\mathrm{K}^{+}$currents underlie firing rate adaptation. $A$, FRA depends on $\mathrm{K}^{+}$currents. The driving force for $\mathrm{K}^{+}$was increased by lowering extracellular $\mathrm{K}^{+}$from $5 \mathrm{~mm}$ (control) to $1 \mathrm{~mm}\left(\right.$ low $\mathrm{K}^{+}$). As shown in the firing response traces, adaptation was larger in low $\mathrm{K}^{+}$, indicating a $\mathrm{K}^{+}$-dependent contribution to FRA. $B$, The slow AHP is mediated by $\mathrm{K}^{+}$currents. Overlap of membrane potential responses to a depolarizing step for control condition (thin line) and in low $\mathrm{K}^{+}$(thick line). Arrows point to the slow AHP, which is increased in low $\mathrm{K}^{+}$. Dashed line indicates $-60 \mathrm{mV}$. C, FRA is not mediated by $\mathrm{Ca}^{2+}$-dependent $\mathrm{K}^{+}$ currents. Firing rate responses are shown for control condition and for bath solution containing zero calcium. Adaptation was not blocked but increased in zero- $\mathrm{Ca}^{2+}$.

was 10-fold stronger in the strongly adapting model neuron than in the weakly adapting model neuron. Figure $9, A 2$ and $B 2$, shows examples of real and modeled neuronal responses to hyperpolarizing steps at two different current amplitudes. The values for the PRF conductances required to reproduce the data differed by almost an order of magnitude between the two neurons shown (A and B), in accordance with the PRF measured experimentally (70 and 10 spikes/sec). At firing rates of $\sim 30$ spikes/sec and higher, an adaptation conductance alone was capable of producing transient increases in firing rate after the offset of a hyperpolarizing stimulus similar to PRF (data not shown). The modeled FRA conductance accumulated to a steady-state level during highfrequency firing, deactivated during the hyperpolarization, and its slow reactivation was seen as a PRF-like increase in firing rate after hyperpolarization. These results show that our model can reproduce the range of PRF and FRA observed in MVN neurons.

To determine the roles of adaptation and rebound conductances in the variations in response phase seen in Figure 5, we assessed the responses of the model neurons to sinusoidal input currents. The results are presented in Figure 9, $A 3$ and $B 3$. The model simulated the actual firing rate waveforms well $\left(R^{2}=0.98\right.$ for neuron $\mathrm{A}$ and $R^{2}=0.99$ for neuron B). As shown in Figure $9 C$, model neuron $\mathrm{A}$ exhibited a phase lead with respect to model neuron B of $11-14^{\circ}$ across all frequencies, similar to the phase difference of $11-15^{\circ}$ seen in the experimental data from the real neurons. In addition, the model reproduced the pattern of phase as a function of frequency observed in the real neurons and in the grouped data shown in Figure 5. Model neurons with fixed spike thresholds showed similar phase differences of 5-21 ${ }^{\circ}$ across frequencies. However, the pattern of phase versus frequency in the fixed-threshold model neurons differed from the real neurons, showing an increasing phase lead versus frequency.

To establish whether conductances responsible for PRF and FRA contribute equally to phase lead, the rebound or the adaptation conductance were each removed from the model in turn. We found that an adaptation conductance alone could produce most of the phase lead and that a model neuron with zero adaptation produced sinusoidal firing responses comparable with neurons with the smallest phase leads. This is shown in Figure $9 D$, where the model output for neuron $\mathrm{A}$ is plotted for three combinations of conductances. Removing the FRA conductance abolished the phase advance (crosses), whereas the output of a model without PRF (open circles) resembled that of the full model, except for an initial deviation in the rising phase of the sine wave. The phase values for the curve without FRA was $1.4^{\circ}$, for curve without PRF was $14.3^{\circ}$, and for the full model was $15.0^{\circ}$. Results from fixed threshold models did not differ qualitatively, producing phases of $-1.6^{\circ}$ without FRA, $20.3^{\circ}$ without PRF, and $22.8^{\circ}$ for both PRF and FRA.

When the simulation was run at higher baseline firing rates to avoid cutoff, the PRF conductance contributed very little to the sinusoidal firing response. In fact, at higher firing rates virtually no change was seen when the PRF conductance was left out of the simulation altogether (data not shown). Further analysis of the activation time course of the simulated conductances during the sinusoidal cycle revealed that the adaptation conductance was maximally activated shortly after the peak in firing rate, whereas the PRF conductance activated maximally during the rising phase of the sinusoidal firing rate.

These findings indicate that the observed phase lead in neurons with strong PRF and FRA was primarily caused by mechanisms underlying adaptation. Thus, whereas FRA and PRF were strongly correlated in MVN neurons, adaptation conductances dictated temporal firing dynamics to a larger extent than did rebound conductances. This is consistent with the pharmacological dissociation of PRF and FRA shown in earlier figures: block of $I_{\mathrm{H}}$ affected PRF, but not FRA and phase leads. Finally, at higher firing rates response phase appears to be entirely governed by FRA. 


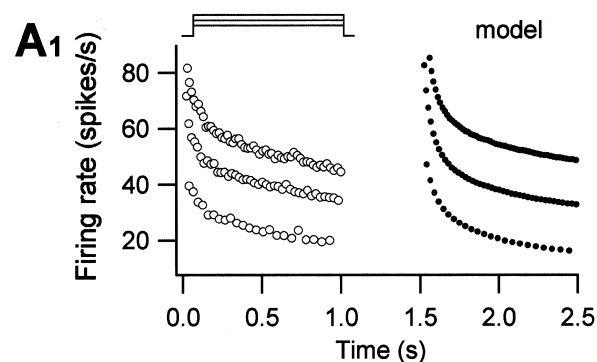

$\mathrm{A}_{2}$

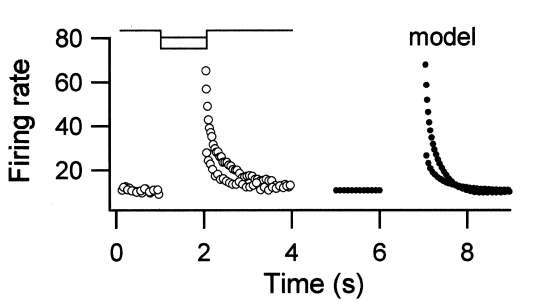

$\mathrm{A}_{3}$

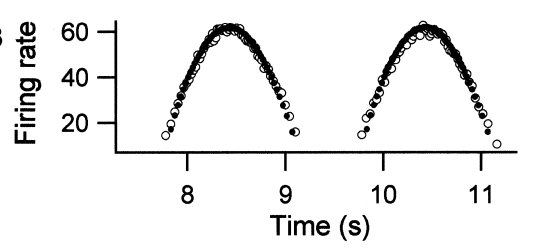

$\mathbf{B}_{1}$

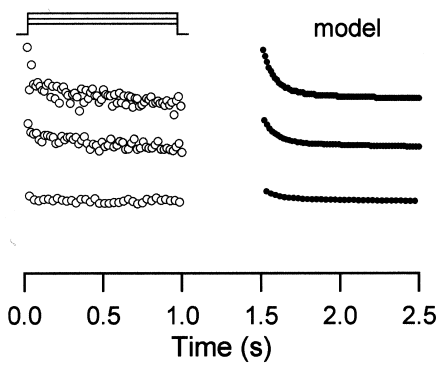

$\mathbf{B}_{2}$
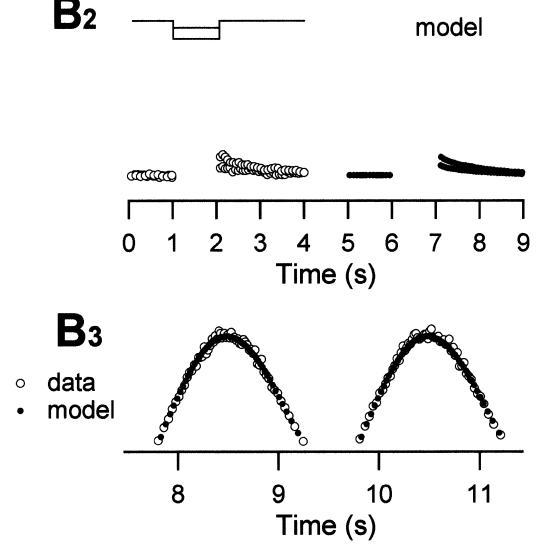
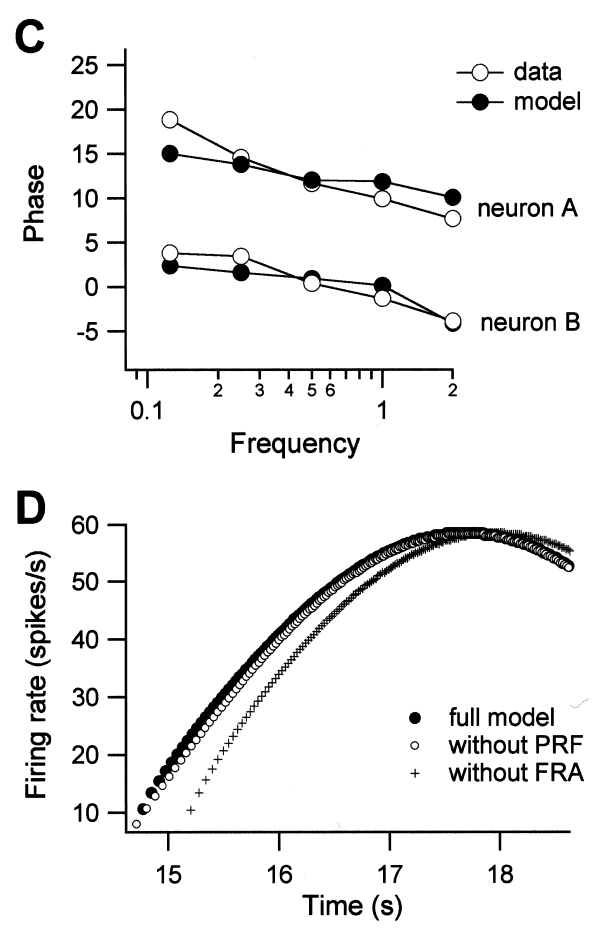

Figure 9. Firing dynamics of an integrate-and-fire model with rebound and adaptation currents. $A 1-A 3$, Firing responses in an $\mathrm{MVN}$ neuron with strong PRF (70 spikes/sec; left) and in a modeled neuron (right). Responses to depolarizing steps (A1), hyperpolarizing steps (A2), and sinusoidal current injection at $0.5 \mathrm{~Hz}(A 3)$ are shown. The simulated adaptation and rebound conductances were adjusted until the model reproduced the data. The model was then tested with sinusoidal current injection without further adjustments of parameters. The response phase of the model $\left(12.1^{\circ}\right)$ closely reproduced the experimental result $\left(11.7^{\circ}\right) \cdot B 1-B 3$, As in $A 1-A 3$, but for a neuron with weak PRF $(10$ spikes/sec). Weak FRA $(B 1)$ and weak PRF $(B 2)$ were modeled by reducing the respective conductances by about an order of magnitude with respect to those in neuron A. The modeled response phase was $0.9^{\circ}$, and the experimental result was $0.4^{\circ}$. C, Comparison of phase versus frequency in real and modeled neurons. $D$, Comparison of results from model neuron A with all conductances ( full model), with the PRF conductance set to 0 (without PRF), and with the FRA conductance set to zero (without FRA). The FRA conductance produces a phase lead, whereas the PRF conductance has little effect on phase.

\section{DISCUSSION}

This study investigated temporal firing dynamics in vestibular nucleus neurons and showed that intrinsic firing responses to hyperpolarizing, depolarizing, and sinusoidally modulated input currents vary continuously across neurons. Large, multipolar neurons tend to have strong postinhibitory rebound firing, pronounced spike frequency adaptation, and in response to sinusoidal inputs, exhibit a phase lead with respect to small, nonadapting neurons that lack rebound firing. Pharmacological and modeling analyses revealed that at least two types of ionic currents confer dynamics onto vestibular nucleus neurons: firing rate adaptation is mediated, at least in part, by a slow potassium current, and the same current, together with $I_{\mathrm{H}}$, appears to mediate rebound firing. Variations in response phase correlate both with rebound firing and with adaptation, but are conferred predominantly by ionic currents that underlie adaptation. These findings imply that the heterogeneity of neuronal firing dynamics observed in intact animals in response to head movement stimuli reflect intrinsic membrane conductances as well as differences in synaptic inputs.

\section{Implications for interpreting response dynamics recorded in vivo}

When challenged with sinusoidally modulating head movements, vestibular nucleus neurons recorded in awake, behaving animals respond with sinusoidal modulations in firing rate that vary widely in response phase with respect to head velocity. Vestibular nerve afferents also exhibit a range of phases in response to head movements (Goldberg and Fernandez, 1971; Keller, 1976; Louie and Kimm, 1976; Ezure et al., 1978; Anastasio et al., 1985; Boyle and Highstein, 1990). These observations, together with an implicit assumption that intrinsic ionic currents do not modify the firing neuronal responses, have been widely used to make inferences about the nature of vestibular nerve inputs onto particular classes of vestibular nucleus neurons (Keller and Precht, 1979; Lisberger and Miles, 1980; Baker et al., 1984; Kasper et al., 1988; Wilson et al., 1990; Endo et al., 1995). Our results, however, indicate that the filtering properties of the spike-generating mechanisms intrinsic to vestibular nucleus neurons are markedly heterogenous and result in differences in firing dynamics across neurons in response to the same stimulus. A recent paper focused on firing response gain has reached a similar conclusion (Ris et al., 2001). This implies that in behaving animals, response dynamics alone cannot be used to infer the nature of synaptic inputs onto vestibular nucleus neurons.

\section{Potential identity of neurons with distinct firing dynamics}

Neurons in the vestibular nuclei are heterogenous with respect to their synaptic inputs and their projection patterns, as well as their function in vestibular and oculomotor processing (Highstein, 1988). The diversity of firing responses to intracellular current injection observed in this study may reflect this heterogeneity. The lack of topographic or layered organization in the vestibular nuclei precludes assigning functional roles to subtypes of neurons recorded in brain slices in the absence of information about neuronal connections. Nonetheless, the following possibilities can 
be put forward based on considerations of neuronal morphology and firing dynamics.

Of the relatively few studies that describe the morphology of vestibular nucleus neurons that have been functionally identified in vivo, two classes of neurons with large multipolar dendrites have been identified: neurons that project to the oculomotor nucleus via the ascending tract of deiters (ATD) (Reisine et al., 1981; Nguyen and Spencer, 1999) and neurons that project to the cerebellar flocculus (Mitsacos et al., 1983). ATD neurons display a phase lead with respect to head velocity (Reisine and Highstein, 1979; Reisine et al., 1981), whereas flocculus-projecting neurons fire in phase with response to head velocity (Cheron et al., 1996). Given the intrinsic phase lead in the large multipolar neurons in our sample, it is plausible that some of them are ATD neurons. As such, intrinsic membrane currents could help these neurons to provide a fast-feedforward signal to the oculomotor nucleus that helps to overcome the sluggishness of the oculomotor plant (Reisine and Highstein, 1979). The large neurons in the rostral MVN that project to the contralateral abducens nucleus (McCrea et al., 1987) would similarly benefit from an intrinsic phase lead.

Postinhibitory rebound firing has been observed in the subset of MVN neurons that receive powerful synaptic inhibition from the cerebellar flocculus [flocculus target neurons (FTNs)] (Kawaguchi, 1985; Sato et al., 1988; du Lac and Lisberger, 1992; Lisberger et al., 1994; Stahl and Simpson, 1995; Zhang et al., 1995). The morphology of FTNs, which constitute a heterogenous population (De Zeeuw and Berrebi, 1995), has been reported only anectodally; however some FTNs appear to have large somata and multipolar dendrites (Kawaguchi, 1985; du Lac and Lisberger, 1992). FTNs have been distinguished from nonFTNs by a pronounced phase lead in their firing rate responses to head movement (Stahl and Simpson, 1995). Although this phase lead has been attributed to Purkinje cell input (Stahl and Simpson, 1995), it is possible that intrinsic membrane currents also play a role and that some of the neurons with strong rebound firing in the present study are FTNs.

\section{lonic currents underlying intrinsic firing dynamics}

A number of ionic currents have been identified previously in MVN neurons. These include calcium and sodium currents responsible for plateau potentials (Serafin et al., 1991b; Johnston et al., 1994), calcium currents involved in rebound responses (Serafin et al., 1990), and calcium-activated potassium currents that influence the AHP and firing response gain (Johnston et al., 1994; Smith et al., 2002). Modeling studies have suggested that variations in potassium currents could account for differences in firing rate adaptation and the patterns of gain versus frequency observed in neurons classified as types A and B (Av-Ron and Vidal, 1999; Ris et al., 2001). Our findings indicate that potassium currents play a role in generating the diversity of firing responses in MVN neurons, but that rather than forming discrete classes, MVN neurons display a continuous distribution of firing properties, including adaptation, postinhibitory rebound firing, and response phase.

Our results indicate that firing response adaptation in $\mathrm{MVN}$ neurons is at least partially mediated by the summation of slow $\mathrm{K}^{+}$currents that are activated during each action potential and progressively hyperpolarize the membrane during repetitive firing. A potassium-dependent AHP followed the decline in firing evoked by sustained depolarization and was well correlated with the extent of adaptation. Two predominant types of slow $\mathrm{K}^{+}$ currents have been shown to be activated during repetitive firing by calcium and sodium influx, respectively. $\mathrm{Ca}^{2+}$-dependent $\mathrm{K}^{+}$ currents are responsible for firing rate adaptation in some neurons (Madison and Nicoll, 1984; Yarom et al., 1985; Yen et al., 1999). However, our data indicate that $\mathrm{Ca}^{2+}$-dependent $\mathrm{K}^{+}$ currents do not underlie adaptation in MVN neurons: removing extracellular calcium abolished neither adaptation nor the slow AHP. An alternative possibility is that $\mathrm{Na}^{+}$-dependent $\mathrm{K}^{+}$currents that are activated by sodium influx during each action potential contribute to adaptation in $\mathrm{MVN}$ neurons. $\mathrm{Na}^{+}$dependent $\mathrm{K}^{+}$currents underlie the slow AHP after spike trains (Kubota and Saito, 1991; Safronov and Vogel, 1996; Kim and McCormick, 1998) and have been implicated in firing rate adaptation in cortical neurons (Schwindt et al., 1989; Sanchez-Vives et al., 2000).

Postinhibitory rebound firing is partially mediated by the hyperpolarization-activated, mixed cationic current $I_{\mathrm{H}}$ in almost all MVN neurons, as it is in many other cell types (Pape, 1996). The voltage dependence of $I_{\mathrm{H}}$ in MVN neurons has not been reported. However in other neurons, $I_{\mathrm{H}}$ is activated with membrane hyperpolarizations below approximately -45 to $-55 \mathrm{mV}$ and shows maximal activation at membrane potentials negative to -110 mV (Pape, 1996). Substantial PRF was observed with membrane hyperpolarizations beyond approximately $-60 \mathrm{mV}$ in the MVN neurons that showed nearly complete block of PRF with $\mathrm{Cs}^{+}$or $\mathrm{ZD} 7288$, consistent with the known activation ranges of $I_{\mathrm{H}^{*}}$.

$I_{\mathrm{H}}$ contributed a variable fraction of rebound firing to MVN neurons. The remaining component required either currents activated during action potentials or subthreshold sodium currents or both, because no rebound depolarization of the membrane remained after a combination of $I_{\mathrm{H}}$ blockade and TTX. Although lack of specific pharmacological antagonists precludes definitive identification, we postulate that the same slow potassium current mediates both firing rate adaptation and the $I_{\mathrm{H}}$-independent component of rebound firing. Our simulations using an integrateand-fire model with an additional spike-dependent cumulative outward current are consistent with this notion. Cumulative activation of an outward current during spontaneous firing would result in a tonic hyperpolarizing drive to MVN neurons. Further hyperpolarization by inputs that reduced firing would reduce the level of this drive, resulting in a transient depolarization during reactivation of the outward current after the offset of the hyperpolarizing stimulus. A shared contribution by slow $\mathrm{K}^{+}$currents could account for the correlation between FRA, PRF, and sinusoidal response phase in $\mathrm{MVN}$ neurons.

\section{REFERENCES}

Aizenman CD, Linden DJ (1999) Regulation of the rebound depolarization and spontaneous firing patterns of deep nuclear neurons in slices of rat cerebellum. J Neurophysiol 82:1697-1709.

Anastasio TJ, Correia MJ, Perachio AA (1985) Spontaneous and driven responses of semicircular canal primary afferents in the unanesthetized pigeon. J Neurophysiol 54:335-347.

Av-Ron E, Vidal PP (1999) Intrinsic membrane properties and dynamics of medial vestibular neurons: a simulation. Biol Cybern 80:383-392.

Azouz R, Jensen MS, Yaari Y (1996) Ionic basis of spike afterdepolarization and burst generation in adult rat hippocampal CA1 pyramidal cells. J Physiol (Lond) 492:211-223.

Babalian A, Vibert N, Assie G, Serafin M, Muhlethaler M, Vidal PP (1997) Central vestibular networks in the guinea-pig: functional characterization in the isolated whole brain in vitro. Neuroscience 81:405-426.

Baker J, Goldberg J, Hermann G, Peterson B (1984) Spatial and temporal response properties of secondary neurons that receive convergent input in vestibular nuclei of alert cats. Brain Res 294:138-143.

Baldissera F, Gustafsson B, Parmiggiani F (1978) Saturating summation of the afterhyperpolarization conductance in spinal motoneu- 
rones: a mechanism for 'secondary range' repetitive firing. Brain Res 146:69-82.

Boyle R, Highstein SM (1990) Resting discharge and response dynamics of horizontal semicircular canal afferents of the toadfish, Opsanus tau. J Neurosci 10:1557-1569.

Brueckner S, Sekirnjak C, Faulstich M, Vissel B, Bollinger J, du Lac S (2001) Cerebellar target neurons in the vestibular nucleus identified using a transgenic approach. Soc Neurosci Abstr 27:298.37.

Buettner UW, Buttner U, Henn V (1978) Transfer characteristics of neurons in vestibular nuclei of the alert monkey. J Neurophysiol 41:1614-1628.

Cheron G, Escudero M, Godaux E (1996) Discharge properties of brain stem neurons projecting to the flocculus in the alert cat. I. Medical vestibular nucleus. J Neurophysiol 76:1759-1774.

De Zeeuw CI, Berrebi AS (1995) Postsynaptic targets of Purkinje cell terminals in the cerebellar and vestibular nuclei of the rat. Eur J Neurosci 7:2322-2333.

Destexhe A, Bal T, McCormick DA, Sejnowski TJ (1996) Ionic mechanisms underlying synchronized oscillations and propagating waves in a model of ferret thalamic slices. J Neurophysiol 76:2049-2070.

du Lac S, Lisberger SG (1992) Eye movements and brainstem neuronal responses evoked by cerebellar and vestibular stimulation in chicks. J Comp Physiol [A] 171:629-638.

du Lac S, Lisberger SG (1995a) Membrane and firing properties of avian medial vestibular nucleus neurons in vitro. J Comp Physiol [A] 176:641-651.

du Lac S, Lisberger SG (1995b) Cellular processing of temporal information in medial vestibular nucleus neurons. J Neurosci 12:8000-8010.

Endo K, Thomson DB, Wilson VJ, Yamaguchi T, Yates BJ (1995) Vertical vestibular input to and projections from the caudal parts of the vestibular nuclei of the decerebrate cat. J Neurophysiol 74:428-436.

Ezure K, Schor RH, Yoshida K (1978) The response of horizontal semicircular canal afferents to sinusoidal rotation in the cat. Exp Brain Res 33:27-39.

Faber ES, Callister RJ, Sah P (2001) Morphological and electrophysiological properties of principal neurons in the rat lateral amygdala in vitro. J Neurophysiol 85:714-723.

Fernandez C, Goldberg J (1971) Physiology of peripheral neurons innervating semicircular canals of the squirrel monkey. II. Response to sinusoidal stimulation and dynamics of peripheral vestibular system. J Neurophysiol 34:661-675.

Fuchs AF, Kimm J (1975) Unit activity in vestibular nucleus of the alert monkey during horizontal angular acceleration and eye movement. J Neurophysiol 38:1140-1161.

Goldberg JM, Fernandez C (1971) Physiology of peripheral neurons innervating semicircular canals of the squirrel monkey. 3. Variations among units in their discharge properties. J Neurophysiol 34:676-684.

Highstein SM (1988) Sensory-to-motor transformations in the vestibular system. Brain Behav Evol 31:25-33.

Huguenard JR (1996) Low-threshold calcium currents in central nervous system neurons. Annu Rev Physiol 58:329-348.

Jahnsen H, Llinas R (1984) Ionic basis for the electro-responsiveness and oscillatory properties of guinea-pig thalamic neurones in vitro. J Physiol (Lond) 349:227-247.

Johnston AR, MacLeod NK, Dutia MB (1994) Ionic conductances contributing to spike repolarization and after-potentials in rat medial vestibular nucleus neurones. J Physiol (Lond) 481:61-77.

Kasper J, Schor RH, Wilson VJ (1988) Response of vestibular neurons to head rotations in vertical planes. I. Response to vestibular stimulation. J Neurophysiol 60:1753-1764.

Kawaguchi Y (1985) Two groups of secondary vestibular neurons mediating horizontal canal signals, probably to the ipsilateral medial rectus muscle, under inhibitory influences from the cerebellar flocculus in rabbits. Neurosci Res 2:434-446.

Keller EL (1976) Behavior of horizontal semicircular canal afferents in alert monkey during vestibular and optokinetic stimulation. Exp Brain Res 24:459-471.

Keller EL, Precht W (1979) Adaptive modification of central vestibular neurons in response to visual stimulation through reversing prisms. J Neurophysiol 42:896-911.

Kim U, McCormick DA (1998) Functional and ionic properties of a slow afterhyperpolarization in ferret perigeniculate neurons in vitro. J Neurophysiol 80:1222-1235.

Knight BW (1972) Dynamics of encoding in a population of neurons. J Gen Physiol 59:734-766.

Kubota M, Saito N (1991) Sodium- and calcium-dependent conductances of neurones in the zebra finch hyperstriatum ventrale pars caudale in vitro. J Physiol (Lond) 440:131-142.

Lisberger SG, Miles FA (1980) Role of primate medial vestibular nucleus in adaptive plasticity of vestibulo-ocular reflex. J Neurophysiol 43:1725-1745.

Lisberger SG, Pavelko TA, Broussard DM (1994) Responses during eye movements of brain stem neurons that receive monosynaptic inhibition from the flocculus and ventral paraflocculus in monkeys. J Neurophysiol $72: 909-927$.
Liu YH, Wang XJ (2001) Spike-frequency adaptation of a generalized leaky integrate-and-fire model neuron. J Comput Neurosci 10:25-45.

Llinas RR (1988) The intrinsic electrophysiological properties of mammalian neurons: insights into central nervous system function. Science 242:1654-1664.

Louie AW, Kimm J (1976) The response of 8th nerve fibers to horizontal sinusoidal oscillation in the alert monkey. Exp Brain Res 24:447-457.

Madison DV, Nicoll RA (1984) Control of the repetitive discharge of rat CA1 pyramidal neurones in vitro. J Physiol (Lond) 354:319-331.

McCrea RA, Strassman A, May E, Highstein SM (1987) Anatomical and physiological characteristics of vestibular neurons mediating the horizontal vestibulo-ocular reflex of the squirrel monkey. J Comp Neurol 264:547-570.

Mitsacos A, Reisine H, Highstein SM (1983) The superior vestibular nucleus: an intracellular HRP study in the cat. II. Non-vestibulo-ocular neurons. J Comp Neurol 215:92-107.

Murphy GJ, du Lac S (2001) Postnatal development of spike generation in rat medial vestibular nucleus neurons. J Neurophysiol 85:1899-1906.

Nguyen LT, Spencer RF (1999) Abducens internuclear and ascending tract of Deiters inputs to medial rectus motoneurons in the cat oculomotor nucleus: neurotransmitters. J Comp Neurol 411:73-86.

Oertel D (1997) Encoding of timing in the brain stem auditory nuclei of vertebrates. Neuron 19:959-962.

Pape HC (1996) Queer current and pacemaker: the hyperpolarizationactivated cation current in neurons. Annu Rev Physiol 58:299-327.

Reisine H, Highstein SM (1979) The ascending tract of Deiters' conveys a head velocity signal to medial rectus motoneurons. Brain Res 170:172-176.

Reisine H, Strassman A, Highstein SM (1981) Eye position and head velocity signals are conveyed to medial rectus motoneurons in the alert cat by the ascending tract of Deiters'. Brain Res 211:153-157.

Ris L, Hachemaoui M, Vibert N, Godaux E, Vidal PP, Moore LE (2001) Resonance of spike discharge modulation in neurons of the guinea pig medial vestibular nucleus. J Neurophysiol 86:703-716.

Safronov BV, Vogel W (1996) Properties and functions of $\mathrm{Na}(+)$ activated $\mathrm{K}+$ channels in the soma of rat motoneurones. J Physio (Lond) 497:727-734.

Sah P, Davies P (2000) Calcium-activated potassium currents in mammalian neurons. Clin Exp Pharmacol Physiol 27:657-663

Sanchez-Vives MV, Nowak LG, McCormick DA (2000) Cellular mechanisms of long-lasting adaptation in visual cortical neurons in vitro. J Neurosci 20:4286-4299.

Sato Y, Kanda K, Kawasaki T (1988) Target neurons of floccular middle zone inhibition in medial vestibular nucleus. Brain Res 446:225-235.

Schwindt PC, Spain WJ, Crill WE (1989) Long-lasting reduction of excitability by a sodium-dependent potassium current in cat neocortical neurons. J Neurophysiol 61:233-244.

Scudder CA, Fuchs AF (1992) Physiological and behavioral identification of vestibular nucleus neurons mediating the horizontal vestibuloocular reflex in trained rhesus monkeys. J Neurophysiol 68:244-264.

Serafin M, Khateb A, de Waele C, Vidal PP, Muhlethaler M (1990) Low threshold calcium spikes in medial vestibular nuclei neurones in vitro: a role in the generation of the vestibular nystagmus quick phase in vivo? Exp Brain Res 82:187-190.

Serafin M, de Waele C, Khateb A, Vidal PP, Muhlethaler M (1991a) Medial vestibular nucleus in the guinea-pig. I. Intrinsic membrane properties in brainstem slices. Exp Brain Res 84:417-425.

Serafin M, de Waele C, Khateb A, Vidal PP, Muhlethaler M (1991b) Medial vestibular nucleus in the guinea-pig. II. Ionic basis of the intrinsic membrane properties in brainstem slices. Exp Brain Re $84: 426-433$.

Shinoda Y, Yoshida K (1974) Dynamic characteristics of responses to horizontal head angular acceleration in vestibuloocular pathway in the cat. J Neurophysiol 37:653-673.

Skavenski AA, Robinson DA (1973) Role of abducens neurons in vestibuloocular reflex. J Neurophysiol 36:724-738.

Smith MR, Nelson AB, du Lac S (2002) Regulation of firing response gain by calcium-dependent mechanisms in vestibular nucleus neurons. $\mathrm{J}$ Neurophysiol, in press.

Stahl JS, Simpson JI (1995) Dynamics of rabbit vestibular nucleus neurons and the influence of the flocculus. J Neurophysiol 73:1396-1413.

Wilson VJ, Yamagata Y, Yates BJ, Schor RH, Nonaka S (1990) Response of vestibular neurons to head rotations in vertical planes. III. Response of vestibulocollic neurons to vestibular and neck stimulation. J Neurophysiol 64:1695-1703.

Yarom Y, Sugimori M, Llinas R (1985) Ionic currents and firing patterns of mammalian vagal motoneurons in vitro. Neuroscience 16:719-737.

Yen JC, Chan JY, Chan SH (1999) Involvement of apamin-sensitive SK channels in spike frequency adaptation of neurons in nucleus tractus solitarii of the rat. J Biomed Sci 6:418-424.

Zhang Y, Partsalis AM, Highstein SM (1995) Properties of superior vestibular nucleus flocculus target neurons in the squirrel monkey. I. General properties in comparison with flocculus projecting neurons. J Neurophysiol 73:2261-2278. 